\title{
Web-Based Interventions Targeting Cardiovascular Risk Factors in Middle-Aged and Older People: A Systematic Review and Meta-Analysis
}

Cathrien RL Beishuizen ${ }^{1}$, MSc, MD; Blossom CM Stephan², PhD; Willem A van Gool ${ }^{1}$, MD, PhD; Carol Brayne ${ }^{3}$, MD; Ron JG Peters ${ }^{4}, \mathrm{MD}, \mathrm{PhD}$; Sandrine Andrieu ${ }^{5}, \mathrm{MD}$, PhD; Miia Kivipelto ${ }^{6}$, MD, PhD; Hilkka Soininen ${ }^{7}, \mathrm{MD}$, $\mathrm{PhD}$; Wim B Busschers ${ }^{8}$, MSc; Eric P Moll van Charante ${ }^{8}, \mathrm{MD}, \mathrm{PhD}$; Edo Richard ${ }^{1,9}, \mathrm{MD}, \mathrm{PhD}$

\footnotetext{
${ }^{1}$ Academic Medical Center, Department of Neurology, University of Amsterdam, Amsterdam, Netherlands

${ }^{2}$ Institute of Health and Society, Newcastle University Institute for Ageing, Newcastle University, Newcastle upon Tyne, United Kingdom

${ }^{3}$ Cambridge Institute of Public Health, Department of Public Health and Primary Care, University of Cambridge, Cambridge, United Kingdom

${ }^{4}$ Academic Medical Center, Department of Cardiology, University of Amsterdam, Amsterdam, Netherlands

${ }^{5}$ Inserm U1027, Department of Epidemiology and Public Health, Toulouse University Hospital, Toulouse, France

${ }^{6}$ Aging Research Center, Alzheimer Disease Research Center, Karolinska Institutet, Stockholm, Sweden

${ }^{7}$ Department of Neurology, University of Eastern Finland and Kuopio University Hospital, Kuopio, Finland

${ }^{8}$ Academic Medical Center, Department of General Practice, University of Amsterdam, Amsterdam, Netherlands

${ }^{9}$ Department of Neurology, Radboud University Medical Center, Nijmegen, Netherlands
}

\section{Corresponding Author:}

Edo Richard, MD, PhD

Academic Medical Center

Department of Neurology

University of Amsterdam

PO Box 22660

Amsterdam, 1100 DD

Netherlands

Phone: 31205663446

Fax: 31205669290

Email: e.richard@amc.uva.nl

\section{Abstract}

Background: Web-based interventions can improve single cardiovascular risk factors in adult populations. In view of global aging and the associated increasing burden of cardiovascular disease, older people form an important target population as well.

Objective: In this systematic review and meta-analysis, we evaluated whether Web-based interventions for cardiovascular risk factor management reduce the risk of cardiovascular disease in older people.

Methods: Embase, Medline, Cochrane and CINAHL were systematically searched from January 1995 to November 2014. Search terms included cardiovascular risk factors and diseases (specified), Web-based interventions (and synonyms) and randomized controlled trial. Two authors independently performed study selection, data-extraction and risk of bias assessment. In a meta-analysis, outcomes regarding treatment effects on cardiovascular risk factors (blood pressure, glycated hemoglobin A1c (HbA1C), low-density lipoprotein (LDL) cholesterol, smoking status, weight and physical inactivity) and incident cardiovascular disease were pooled with random effects models.

Results: A total of 57 studies $(\mathrm{N}=19,862)$ fulfilled eligibility criteria and 47 studies contributed to the meta-analysis. A significant reduction in systolic blood pressure (mean difference $-2.66 \mathrm{mmHg}, 95 \% \mathrm{CI}-3.81$ to -1.52 ), diastolic blood pressure (mean difference $-1.26 \mathrm{mmHg}, 95 \% \mathrm{CI}-1.92$ to -0.60 ), HbA1c level (mean difference $-0.13 \%, 95 \% \mathrm{CI}-0.22$ to -0.05 ), LDL cholesterol level (mean difference $-2.18 \mathrm{mg} / \mathrm{dL}, 95 \% \mathrm{CI}-3.96$ to -0.41 ), weight (mean difference $-1.34 \mathrm{~kg}$, 95\% CI -1.91 to -0.77 ), and an increase of physical activity (standardized mean difference $0.25,95 \%$ CI 0.10-0.39) in the Web-based intervention group was found. The observed effects were more pronounced in studies with short ( $<12$ months) follow-up and studies that combined the Internet application with human support (blended care). No difference in incident cardiovascular disease was found between groups (6 studies). 
Conclusions: Web-based interventions have the potential to improve the cardiovascular risk profile of older people, but the effects are modest and decline with time. Currently, there is insufficient evidence for an effect on incident cardiovascular disease. A focus on long-term effects, clinical endpoints, and strategies to increase sustainability of treatment effects is recommended for future studies.

(J Med Internet Res 2016;18(3):e55) doi: 10.2196/jmir.5218

\section{KEYWORDS}

eHealth; cardiovascular disease; prevention; older people; aging; systematic review; meta-analysis

\section{Introduction}

The field of eHealth is expanding the potential of contemporary medicine [1]. Global aging and its associated burden of cardiovascular disease may expand the scope for innovative Internet interventions [2,3]. Current cardiovascular risk management programs in primary care will become too expensive and, although they are highly effective in research settings [4-6], their effectiveness is markedly lower in daily life [7]. This evidence-practice gap has several causes [8]. Adherence to life-long lifestyle and medication regimens is a serious challenge, illustrated by long-term adherence rates in chronic diseases that average as low as 50\% [9,10]. Web-based interventions are cheap, have a wide reach, and they enable self-management [11]. This renders Web-based interventions potentially powerful and scalable tools to enhance sustained adherence in cardiovascular risk management [12].

Older people form an important target population because cardiovascular risk reduction appears effective until old age [13-16]. In 2012, 42\% of European people aged between 55 and 74 years used the Internet and this number is increasing [17]. Meta-analyses showed that Web-based interventions targeting single cardiovascular risk factors can induce improvements in adult populations [18-21]. However, optimal cardiovascular prevention and risk management practice, as affirmed by the European Society of Cardiology [22] and the American Heart Association [23], requires targeting the complete cardiovascular risk profile. This is particularly applicable for older people, who often have multiple risk factors or already suffered a cardiovascular event. A comprehensive approach would increase the value of Web-based interventions for daily practice. Currently, little is known about the effectiveness of Web-based interventions in older people.

In this systematic review and meta-analysis, we aim to answer the question whether Web-based interventions for cardiovascular risk factor management reduce cardiovascular risk and disease in older people.

\section{Methods}

\section{Search Strategy and Selection of Eligible Studies}

We performed a systematic literature search for randomized controlled trials (RCT) on Web-based interventions in older people targeting one or more cardiovascular risk factors and/or disease. Methods were predefined in a research protocol using the PRISMA checklist and the Systematic Reviews Guidelines of the Center of Reviews and Dissemination (Multimedia Appendix 1). We defined Web-based interventions as
Web-based participant-centered treatment or prevention programs delivered via the Internet and interacting with the participant in a tailored fashion [24,25]. Internet had to be the main medium through which the intervention was delivered, but other media (phone, face-to-face) could be included too. We excluded the following eHealth interventions: telemonitoring, telemedicine, and mobile phone-mediated interventions. The target of the intervention had to be one or more cardiovascular risk factors and/or cardiovascular disease. Thus, we included interventions for both primary and secondary prevention of cardiovascular disease [22]. The target population had to have a mean age of 50 years or older and could have a mixed level of cardiovascular risk (one or more cardiovascular risk factors or established cardiovascular disease).

Main outcomes of interest were incident cardiovascular disease (myocardial infarction, angina pectoris, heart failure, stroke or transient ischemic attack, and peripheral arterial disease), cardiovascular mortality and overall mortality, and changes in cardiovascular risk factors including blood pressure (BP), glycated hemoglobin $\mathrm{A}_{1 \mathrm{c}}\left(\mathrm{HbA}_{1 \mathrm{c}}\right)$, low-density lipoprotein (LDL) cholesterol, smoking status, weight, level of physical exercise, or a composite cardiovascular risk score.

We performed a comprehensive literature search in the EMBASE, Medline, CINAHL, and Cochrane databases from 1995 onward (because the Internet was not widely available before then). Key search terms were cardiovascular risk factors and diseases (separate diseases and risk factors specified), terms related to aspects of cardiovascular risk management (eg, diet, exercise, BP control), Web-based interventions (including all definitions and synonyms), and RCT/review/meta-analysis. The search was last updated on November 3, 2014 by CRB. The comprehensive search strategy is provided in Multimedia Appendix 2. Studies were included if (1) they were on Web-based interventions targeting cardiovascular risk factors and/or disease, (2) study design was a RCT, (3) at least 50 patients were included, (4) mean age was at least 50 years, (5) the duration of the intervention was 4 or more weeks and follow-up was 3 or more months, (6) at least one of the outcomes of our interest was reported, and (7) language was English. Study selection was performed by two independent researchers (CRB and BS) by means of screening of titles and abstracts, and thereafter reading full texts on the basis of the inclusion criteria. If two publications described the same trial, the paper that reported the primary outcomes of the trial was included. Disagreements were resolved by discussion or by a third investigator (ER). We assessed reviews and meta-analyses encountered with our search strategy to check for additional relevant articles. 


\section{Data Extraction}

Two reviewers (BS and CRB) extracted data using a predefined data extraction form (Multimedia Appendix 3) for half of the included articles and checked each other's results. Extracted information included study characteristics, patient baseline characteristics, characteristics of the intervention and control conditions, and available data on clinical and intermediate outcomes. For BP, glucose control, weight, lipids, and physical activity level, we extracted all baseline and follow-up levels, change scores or mean differences. Corresponding authors were contacted if needed. We used an adapted Cochrane Risk of Bias Tool to evaluate randomization procedures, representativeness of study populations, blinding of outcome assessors (blinding of participants was usually not possible due to study design), completeness of outcome data, and completeness of reporting.

\section{Meta-Analysis}

For categorical variables, we calculated odds ratios with $95 \%$ confidence intervals. We estimated pooled odds ratios with Mantel-Haenszel random-effects models. For continuous outcomes, mean differences or standardized mean differences (Hedges' $g$ effect sizes) with $95 \%$ confidence intervals were calculated. We estimated pooled effects with DerSimonian and Laird random-effects models. All $\mathrm{HbA}_{1 \mathrm{c}}$ values were converted to percentages. All LDL cholesterol values were converted to $\mathrm{mg} / \mathrm{dL}$. All weight values were converted to $\mathrm{kg}$. For level of physical activity, which was assessed with various instruments, we calculated standardized mean differences and 95\% confidence intervals. If mean differences or standardized mean differences were reported, we included them directly in the pooled analyses. If not, we calculated change scores (difference between baseline and follow-up within group) or values assessed at follow-up. If values were measured at multiple time points, we used the values recorded at the last follow-up contact.

For studies with multiple arms, we included only one intervention arm in the meta-analysis in order not to create "unit-of-analysis" error by double counting the control group. Where possible, we selected the Internet-only intervention arm. No data were imputed.

We estimated pooled effects for all single cardiovascular risk factors. To address the overall question of efficacy of Web-based interventions for cardiovascular risk factor management, we evaluated the effect on cardiovascular composite scores, clinical outcomes (cardiovascular morbidity and mortality), and pooled the standardized primary outcomes of all studies. We used the primary outcomes as defined by the authors of the studies.

Funnel plots were inspected to assess for potential publication bias. Statistical heterogeneity was assessed using $\mathrm{Q}$ and $\mathrm{I}^{2}$ tests.
We explored reasons for heterogeneity by jackknife analysis and subgroup analyses. We assessed the following factors in subgroup analyses: study duration (predefined, short term $[<12$ months] versus long term [ $\geq 12$ months]), type of cardiovascular prevention (primary versus secondary) [22], and type of intervention (Internet only or "blended" [Internet application combined with human support]). Subgroup analyses were performed on the studies used for the analysis on primary outcomes only. The latter subgroup analysis (on type of intervention) consisted of two separate analyses, one to evaluate the Internet-only interventions versus the control conditions and one to evaluate the blended interventions versus control conditions. In case a study tested both types of interventions with a multiple-arm design, the appropriate arm was included for each analysis. In addition, we performed a mixed effects meta-regression using the unrestricted maximum likelihood method to explore the association between study duration and effect size (standardized primary outcome). Last, we performed sensitivity analyses for the different domains of the risk-of-bias assessment by repeating the analysis on standardized primary outcomes in subgroups of studies with low risk of bias versus studies with an unclear or high risk of bias. For this analysis, we wanted to include all studies that contributed to one of the meta-analyses. Therefore, we complemented the sample of studies with defined primary outcomes that were cardiovascular risk factors of interest with studies that had not defined their primary outcome. If there was no defined primary outcome, we used the cardiovascular risk factor that was targeted most directly in the intervention studied. We used Review Manager 5.2 to draw the risk-of-bias assessment figure and to calculate standard deviations or $95 \%$ confidence intervals in cases where only standard errors were available in the original data. We used Microsoft Office Excel version 10, SPSS version 20, and Comprehensive Meta Analysis version 2.2.064 for the statistical analyses.

\section{Results}

\section{Study Selection}

The search yielded 5251 papers after removal of duplicates. We did not identify additional studies by searching reference lists. After screening of titles and abstracts, 462 papers remained. Review of these full texts resulted in 57 RCTs (corresponding with 84 papers) that fulfilled the selection criteria and were included in the systematic review. We contacted 16 authors to request additional data: nine authors responded and three authors complied with our request. Out of this final selection, 47 studies could be included in the meta-analysis (see Figure 1 for PRISMA flowchart). 
Figure 1. Prisma flowchart illustrating literature search.

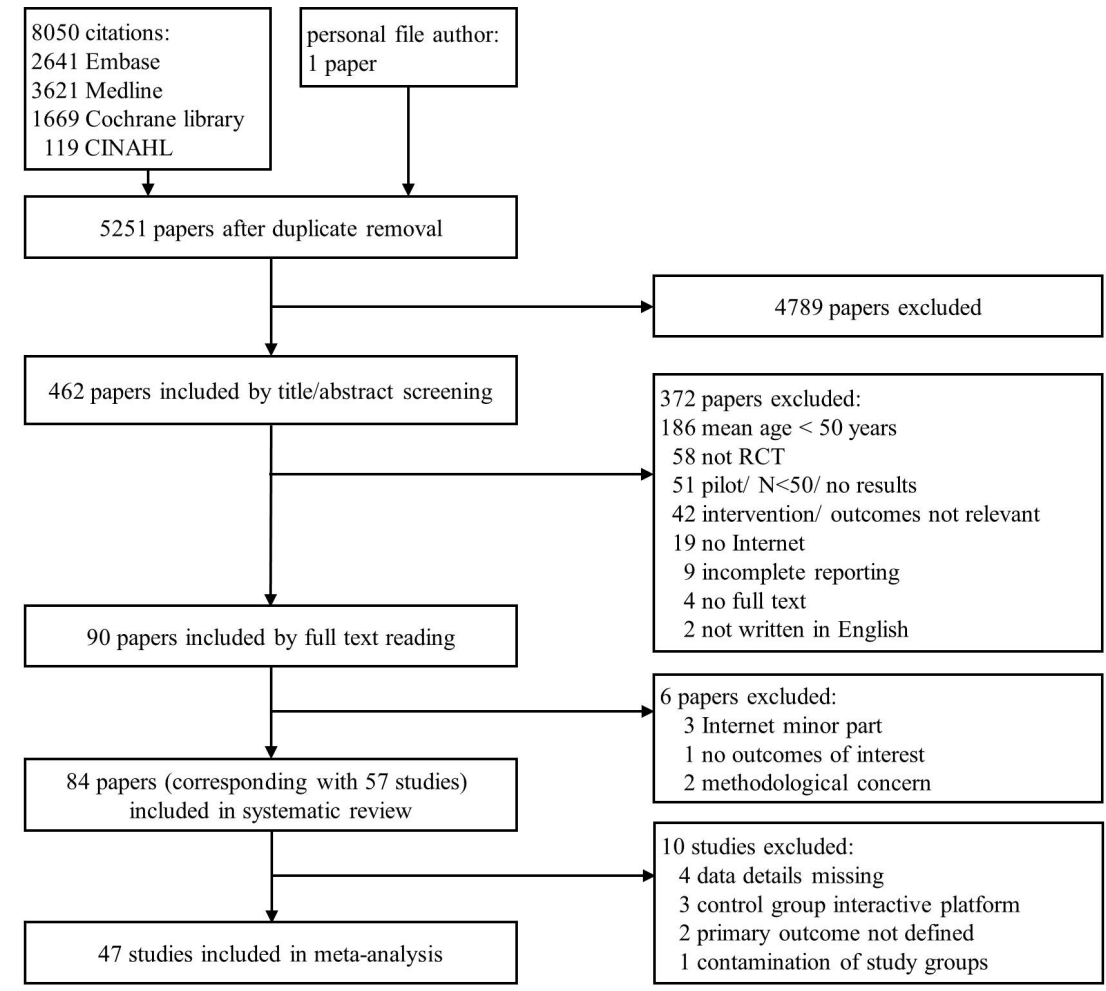

\section{Study Characteristics}

The 57 RCTs included 19,862 individuals (Tables 1-5). Study sample size ranged from 61 to 2140 participants. Median study duration was 9 months (interquartile range [IQR] 6, range 3-60 months). The mean dropout rate was $15 \%$ (range $0 \%-62 \%$ ). The mean age of the study populations ranged from 50 to 71 years. In only 7 studies were all participants older than 50 years of age. All participants had an increased risk of cardiovascular disease: 46 studies conducted primary prevention (control of cardiovascular risk factors or diabetes) and 11 studies conducted secondary prevention. In 41 studies, the intervention targeted a single cardiovascular risk factor; in 16 studies, multiple risk factors were addressed. We found no studies on interventions for smoking cessation meeting our inclusion criteria. In most studies, the primary outcome was change in a specific cardiovascular risk factor targeted by the intervention. Sixteen studies reported on clinical outcomes including new cardiovascular events [26-31] and mortality rates [29-41] as a part of adverse event monitoring. All interventions included lifestyle education and were participant-centered. Forty-four studies stimulated self-management by means of goal setting and self-monitoring. Half of interventions were stand-alone Internet platforms and the other half were "blended" (ie, the platforms were supported by a nurse or another health care professional). Intervention usage was reported by 22 studies. The median percentage of participants logging in to the intervention platform was $72 \%$ (range 33\%-100\%).

\section{Quality Assessment}

Methodological quality of the included studies varied (Multimedia Appendix 4). Most studies adequately described the randomization and allocation concealment procedures. Due to the nature of the interventions, none of the studies had a double-blind design. In 20 studies, outcome assessors were blinded [27,29,30,32,34-40,42,52,54,57,59,61,64,67,81], in 19 studies blinding was not mentioned or unclear [28,31,33,41,43,44,46,48,53,56,60,63,65,69,71,74,76,77,80], and in 18 studies outcome assessors were not blinded [26,45,47,49-51,55,58,62,66,68,70,72,73,75,78,79,82].

\section{Effect of Web-Based Interventions on Single Risk Factors}

Of the 57 studies included in the systemic review, 47 studies [26-32,34-42,44-53,55-60,62,64-68,70,73,74,76-79,81,82] provided sufficient information to be included in the meta-analysis. The mean age of the study populations of these 46 studies had the same range as the complete sample of 57 studies.

\section{Systolic and Diastolic Blood Pressure}

The pooled analysis showed a significant reduction in both systolic and diastolic BP favoring the intervention (26 studies; $\mathrm{n}=7720$; Figures 2 and 3). For systolic BP, the weighted mean difference was $-2.66 \mathrm{mmHg}\left(95 \% \mathrm{CI}-3.81\right.$ to $\left.-1.52 ; \mathrm{I}^{2}=53 \%\right)$. For diastolic BP, the weighted mean difference was -1.26 $\mathrm{mmHg}\left(95 \% \mathrm{CI}-1.92\right.$ to $\left.-0.60 ; \mathrm{I}^{2}=46 \%\right)$. 
Table 1. Characteristics of the studies included for the systematic review: interventions targeting diabetes. ${ }^{\mathrm{a}}$

\begin{tabular}{|c|c|c|c|c|c|c|c|}
\hline Study & $\begin{array}{l}\text { Setting and } \\
\text { study } \\
\text { length }\end{array}$ & Participants & $\begin{array}{l}\text { Age (years), } \\
\text { mean (SD) }\end{array}$ & $\begin{array}{l}\text { Sex }(\% \\
\text { female })\end{array}$ & Intervention & Control & $\begin{array}{l}\text { Primary; sec- } \\
\text { ondary out- } \\
\text { comes }\end{array}$ \\
\hline $\begin{array}{l}\text { Bond } \\
2010[42]\end{array}$ & $\begin{array}{l}\text { 2-arm } \\
\text { RCT; } \\
\text { USA; } 6 \text { m }\end{array}$ & $\begin{array}{l}62 \text { people with DM via } \\
\text { university/veteran clinic }\end{array}$ & $67.2(6.0)$ & 45 & $\begin{array}{l}\text { Website: education, self- } \\
\text { monitoring (glucose, exercise, } \\
\text { weight, BP, medication), fo- } \\
\text { rum; nurse support (email, } \\
\text { chat) }\end{array}$ & $\begin{array}{l}\text { Standard diabetes } \\
\text { care }\end{array}$ & $\begin{array}{l}\mathrm{HbA}_{1 \mathrm{c}}, \mathrm{BP}, \\
\text { weight, total } \\
\text { cholesterol, } \\
\text { HDL choles- } \\
\text { terol }\end{array}$ \\
\hline $\begin{array}{l}\text { IDEA- } \\
\text { TEL } \\
2000- \\
2010[34]\end{array}$ & $\begin{array}{l}\text { 2-arm } \\
\text { RCT; } \\
\text { USA; } 60 \mathrm{~m}\end{array}$ & $\begin{array}{l}1665 \text { Medicare recipients } \\
\text { with DM }\end{array}$ & $70.9(6.7)$ & 63 & $\begin{array}{l}\text { Online home telemedicine } \\
\text { unit: nurse support (video } \\
\text { chat), Web portal for self- } \\
\text { monitoring (glucose, BP), ed- } \\
\text { ucation }\end{array}$ & $\begin{array}{l}\text { Standard diabetes } \\
\text { care }\end{array}$ & $\begin{array}{l}\mathrm{HbA}_{1 \mathrm{c}}, \text { systolic } \\
\mathrm{BP}, \text { diastolic } \\
\mathrm{BP} \text {, total } \\
\text { cholesterol, } \\
\text { LDL choles- } \\
\text { terol }\end{array}$ \\
\hline $\begin{array}{l}\text { D-net } \\
2001[43]\end{array}$ & $\begin{array}{l}\text { 4-arm } \\
\text { RCT; } \\
\text { USA; } 10 \mathrm{~m}\end{array}$ & $\begin{array}{l}320 \text { people with DM2, } \\
\text { Internet, from } 16 \text { GPs }\end{array}$ & $59(9.2)$ & 53 & $\begin{array}{l}\text { Website: (1) Self-manage- } \\
\text { ment (glucose), coach sup- } \\
\text { port; (2) education, forum; ( } 3) \\
1 \text { and } 2 \text { combined }\end{array}$ & $\begin{array}{l}\text { (4) Information on } \\
\text { medical and lifestyle } \\
\text { aspects of diabetes }\end{array}$ & $\begin{array}{l}\text { Not defined; be- } \\
\text { havioral, biolog- } \\
\text { ical, and psy- } \\
\text { chosocial out- } \\
\text { comes }\end{array}$ \\
\hline $\begin{array}{l}\text { My path } \\
2010[44]\end{array}$ & $\begin{array}{l}\text { 3-arm } \\
\text { RCT; } \\
\text { USA; } 12 \mathrm{~m}\end{array}$ & $\begin{array}{l}463 \text { Medicare recipients } \\
\text { with DM } 2, \mathrm{BMI} \geq 25 \\
\mathrm{~kg} / \mathrm{m}^{2} \text { or } \geq 1 \mathrm{CV} \text { risk fac- } \\
\text { tor, Internet }\end{array}$ & $58.4(9.2)$ & 50 & 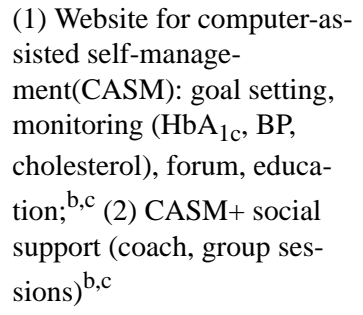 & $\begin{array}{l}\text { (3) Computer-based } \\
\text { health risk appraisal, } \\
\text { no key features of } \\
\text { CASM }\end{array}$ & $\begin{array}{l}\text { Behavior } \\
\text { changes in diet, } \\
\text { physical activi- } \\
\text { ty, medication } \\
\text { adherence }\end{array}$ \\
\hline $\begin{array}{l}\text { My care } \\
\text { team } \\
2005[45]\end{array}$ & $\begin{array}{l}\text { 2-arm } \\
\text { RCT; } \\
\text { USA; } 12 \mathrm{~m}\end{array}$ & $\begin{array}{l}104 \text { people with } \mathrm{DM} \text {, } \\
\mathrm{HbA}_{1 \mathrm{c}} \geq 9.0 \% \text { via veteran } \\
\text { clinic }\end{array}$ & $63.5(7.0)$ & 0.5 & $\begin{array}{l}\text { Website: self-management } \\
\text { (glucose, BP), education, re- } \\
\text { minders (phone); care manag- } \\
\text { er support }\end{array}$ & $\begin{array}{l}\text { DM self-manage- } \\
\text { ment training, usual } \\
\text { care }\end{array}$ & $\begin{array}{l}\mathrm{HbA}_{1 \mathrm{c}} \text { and } \mathrm{BP} \\
\text { at } 3,6,9 \text {, and } \\
12 \mathrm{~m}\end{array}$ \\
\hline $\begin{array}{l}\text { Mobile } \\
\text { DM } 2011 \\
{[32]}\end{array}$ & $\begin{array}{l}\text { 4-arm clus- } \\
\text { ter RCT; } \\
\text { USA; } 12 \mathrm{~m}\end{array}$ & $\begin{array}{l}26 \text { physician practices } \\
\text { with } 163 \text { people with } \mathrm{DM} \\
\text { and } \mathrm{HbA}_{1 \mathrm{c}} \geq 7.5 \%\end{array}$ & $52.8(8.1)$ & 50 & $\begin{array}{l}\text { (2) Self-management via } \\
\text { website + mobile phone, pa- } \\
\text { tient informs doctor; }^{b} \text { (3) } 2+ \\
\text { doctor access to data; (4) } 3+ \\
\text { advice from doctor }^{c}\end{array}$ & (1) Care as usual & $\begin{array}{l}\text { Change in } \\
\mathrm{HbA}_{1 \mathrm{c}} \text { over } 1 \\
\text { year }\end{array}$ \\
\hline $\begin{array}{l}\text { Avdal } \\
2011[46]\end{array}$ & $\begin{array}{l}\text { 2-arm } \\
\text { RCT; } \\
\text { Turkey; } 6 \\
\text { m }\end{array}$ & $\begin{array}{l}122 \text { people with DM2, } \\
\text { Internet from clinic }\end{array}$ & $51(7.3)$ & 51 & $\begin{array}{l}\text { Website: review risk profile, } \\
\text { messaging to researcher, daily } \\
\text { glucose monitoring }\end{array}$ & $\begin{array}{l}\text { Education and usual } \\
\text { care }\end{array}$ & $\begin{array}{l}\mathrm{HbA}_{1 \mathrm{c}} \text {, atten- } \\
\text { dance rates at } \\
\text { outpatient clinic }\end{array}$ \\
\hline $\begin{array}{l}\text { Cho } 2006 \\
{[47]}\end{array}$ & $\begin{array}{l}\text { 2-arm } \\
\text { RCT; } \\
\text { South Ko- } \\
\text { rea; } 30 \mathrm{~m}\end{array}$ & $\begin{array}{l}80 \text { people with } \mathrm{DM} \text {, Inter- } \\
\text { net from clinic }\end{array}$ & $53(9)$ & 39 & $\begin{array}{l}\text { Website: monitoring (glucose, } \\
\text { medication, BP, weight, } \\
\text { lifestyle), nurse feedback, } \\
\text { medication alterations }\end{array}$ & $\begin{array}{l}\text { Conventional note- } \\
\text { keeping record sys- } \\
\text { tem }\end{array}$ & $\begin{array}{l}\mathrm{HbA}_{1 \mathrm{c}} \text { and } \\
\mathrm{HbA}_{1 \mathrm{c}} \text { fluctua- } \\
\text { tion index }\end{array}$ \\
\hline $\begin{array}{l}\text { Lorig } \\
2010[48]\end{array}$ & $\begin{array}{l}\text { 3-arm } \\
\text { RCT; } \\
\text { USA; } 6 \text { m }\end{array}$ & $\begin{array}{l}761 \text { people with } \mathrm{DM} 2 \text {, } \\
\text { Internet }\end{array}$ & $54.3(9.9)$ & 73 & $\begin{array}{l}\text { Self-management website } \\
\text { with peer support: lessons, } \\
\text { action plans, bulletin board, } \\
\text { messaging }\end{array}$ & Care as usual & $\begin{array}{l}\mathrm{HbA}_{1 \mathrm{C}} \text { level at } \\
6 \text { and } 18 \\
\text { months }\end{array}$ \\
\hline $\begin{array}{l}\text { Grant } \\
2008[49]\end{array}$ & $\begin{array}{l}\text { 2-arm clus- } \\
\text { ter RCT; } \\
\text { USA; } 12 \mathrm{~m}\end{array}$ & $\begin{array}{l}244 \text { people with DM, } \\
\mathrm{HbA}_{1 \mathrm{c}}>7.0 \% \text { from } 11 \\
\text { primary clinics }\end{array}$ & $56.1(11.6)$ & 49 & $\begin{array}{l}\text { Online personal health record: } \\
\text { education, diabetes care plan, } \\
\text { agenda, messaging, prescrip- } \\
\text { tion refills }\end{array}$ & $\begin{array}{l}\text { Access to general } \\
\text { website Patient } \\
\text { Gateway }\end{array}$ & $\begin{array}{l}\text { Changes in } \\
\mathrm{HbA}_{1 \mathrm{c}}, \mathrm{BP} \text {, and } \\
\text { LDL choles- } \\
\text { terol }\end{array}$ \\
\hline $\begin{array}{l}\text { McMa- } \\
\text { hon } 2012 \\
{[50]}\end{array}$ & $\begin{array}{l}\text { 3-arm } \\
\text { RCT; } \\
\text { USA; } 12 \text { m }\end{array}$ & $\begin{array}{l}151 \text { people with } \mathrm{DM}, \\
\mathrm{HbA}_{1 \mathrm{c}}>8.5 \% \text { from veter- } \\
\text { an health services }\end{array}$ & $60.2(10.8)$ & 5 & $\begin{array}{l}\text { (1) Self-monitoring via phone } \\
\text { (BP, glucose); (2) website: } \\
\text { self-monitoring (BP, glucose), } \\
\text { education, support by care } \\
\text { managers }{ }^{\text {b,c }}\end{array}$ & $\begin{array}{l}\text { (3) Website with } \\
\text { links to other DM } \\
\text { websites; usual care }\end{array}$ & $\begin{array}{l}\text { Change in } \\
\mathrm{HbA}_{1 \mathrm{c}} \text { and } \mathrm{BP} \\
\text { over time }\end{array}$ \\
\hline
\end{tabular}




\begin{tabular}{|c|c|c|c|c|c|c|c|}
\hline Study & $\begin{array}{l}\text { Setting and } \\
\text { study } \\
\text { length }\end{array}$ & Participants & $\begin{array}{l}\text { Age (years), } \\
\text { mean (SD) }\end{array}$ & $\begin{array}{l}\text { Sex }(\% \\
\text { female })\end{array}$ & Intervention & Control & $\begin{array}{l}\text { Primary; sec- } \\
\text { ondary out- } \\
\text { comes }\end{array}$ \\
\hline $\begin{array}{l}\text { Ralston } \\
2009[51]\end{array}$ & $\begin{array}{l}2 \text {-arm } \\
\text { RCT; } \\
\text { USA; } 12 \mathrm{~m}\end{array}$ & $\begin{array}{l}83 \text { people with } \mathrm{DM} 2 \text {, } \\
\mathrm{HbA}_{1 \mathrm{c}} \geq 7.0 \% \text { and Inter- } \\
\text { net from clinic: } 65 \% \text { with } \\
2 \mathrm{CV} \text { risk factors }\end{array}$ & $57.3(-)$ & 52 & $\begin{array}{l}\text { Electronic medical record: } \\
\text { self-monitoring (glucose, ex- } \\
\text { ercise, diet, medication), sup- } \\
\text { port by care manager, usual } \\
\text { care visits }\end{array}$ & Usual care visits & $\begin{array}{l}\text { Change in } \\
\mathrm{HbA}_{1 \mathrm{c}}\end{array}$ \\
\hline $\begin{array}{l}\text { Kwon } \\
2004 \text { [52] }\end{array}$ & $\begin{array}{l}2 \text {-arm } \\
\text { RCT; } \\
\text { South Ko- } \\
\text { rea; } 3 \mathrm{~m}\end{array}$ & $\begin{array}{l}110 \text { people with } \mathrm{DM} 2 \text {, } \\
\text { Internet from clinic: } 27 \% \\
\text { hypertension }\end{array}$ & $54.1(9.1)$ & 33 & $\begin{array}{l}\text { Website: self-monitoring } \\
\text { (glucose), reminders, profes- } \\
\text { sor/nurse/dietician-support }\end{array}$ & $\begin{array}{l}\text { Monthly visit to dia- } \\
\text { betes specialist }\end{array}$ & $\mathrm{HbA}_{1 \mathrm{c}}$ \\
\hline $\begin{array}{l}\text { EMPOW- } \\
\text { ER-D } \\
2013 \text { [39] }\end{array}$ & $\begin{array}{l}2 \text {-arm } \\
\text { RCT; } \\
\text { USA; } 12 \mathrm{~m}\end{array}$ & $\begin{array}{l}415 \text { people with DM and } \\
\mathrm{HbA}_{1 \mathrm{c}} \geq 7.5 \% \text { from clinic }\end{array}$ & $53.7(10.2)$ & 40 & $\begin{array}{l}\text { Online health record: risk esti- } \\
\text { mation, self-monitoring (glu- } \\
\text { cose, diet, exercise, BP), } \\
\text { nurse support, own doctor in- } \\
\text { formed }\end{array}$ & Usual care & $\mathrm{HbA}_{1 \mathrm{c}}$ at $12 \mathrm{~m}$ \\
\hline $\begin{array}{l}\text { RE- } \\
\text { DEEM } \\
2013[53]\end{array}$ & $\begin{array}{l}\text { 3-arm } \\
\text { RCT; } \\
\text { USA; } 12 \mathrm{~m}\end{array}$ & $\begin{array}{l}392 \text { people with } \mathrm{DM} 2 \text {, } \\
\text { Internet from community } \\
\text { centers }\end{array}$ & $56.1(9.6)$ & 54 & $\begin{array}{l}\text { (1) CASM website: goal set- } \\
\text { ting; self-monitoring }\left(\mathrm{HbA}_{1 \mathrm{c}} \text {, }\right. \\
\mathrm{BP} \text {, cholesterol); } 8 \text { phone } \\
\text { calls; }{ }^{\mathrm{b}} \text { (2) Computer-assisted } \\
\text { self-management + problem } \\
\text { solving treatment (CASP): } \\
\text { CASM + } 8 \text { sessions problem } \\
\text { solving }\end{array}$ & $\begin{array}{l}\text { Computer health risk } \\
\text { appraisal, education, } \\
\text { same phone calls as } \\
\text { intervention }\end{array}$ & $\begin{array}{l}\text { Diabetes dis- } \\
\text { tress; } \mathrm{HbA}_{1 \mathrm{c}} \\
\text { physical activi- } \\
\text { ty, medication } \\
\text { compliance }\end{array}$ \\
\hline
\end{tabular}

a Abbreviations: BP: blood pressure; CASM: computer-assisted self-management; CASP: computer-assisted self-management + problem solving treatment; CV: cardiovascular; DM: diabetes mellitus; DM2: type 2 diabetes mellitus; GP: general practitioner; HbA1c; glycated hemoglobin A1c; HDL: high-density lipoprotein; LDL: low-density lipoprotein.

${ }^{\mathrm{b}}$ For studies with more than 2 arms, this arm was used for all analyses.

${ }^{\mathrm{c}}$ For studies with more than 2 arms, this arm was used for the subgroup analysis on blended interventions.

Figure 2. Effect on systolic blood pressure (26 studies).

\section{Systolic blood pressure $(\mathrm{mmHg})$}

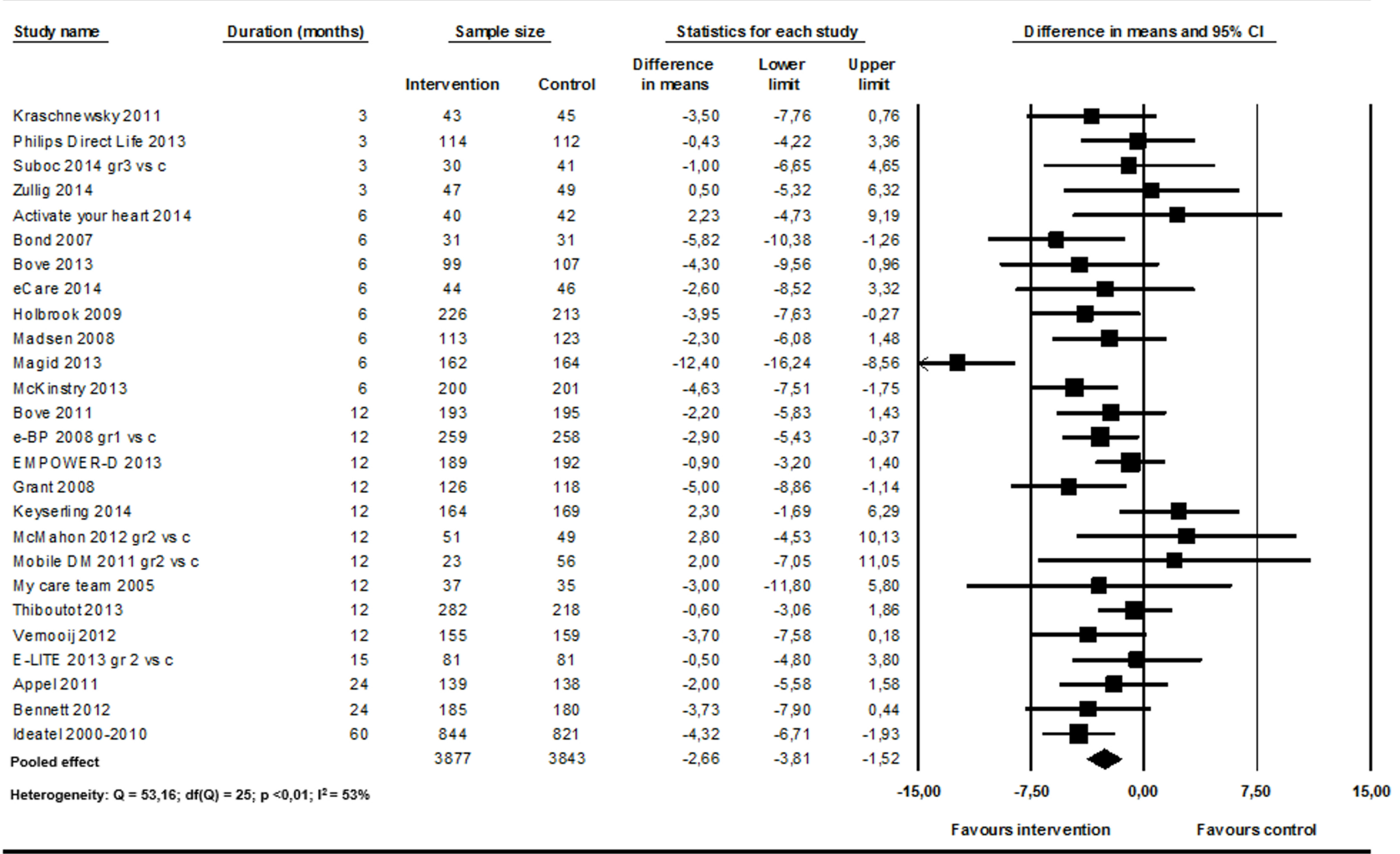


Table 2. Characteristics of the studies included for the systematic review: interventions targeting blood pressure. ${ }^{\text {a }}$

\begin{tabular}{|c|c|c|c|c|c|c|c|}
\hline Study & $\begin{array}{l}\text { Setting and } \\
\text { study } \\
\text { length }\end{array}$ & Participants & $\begin{array}{l}\text { Age (years), } \\
\text { mean (SD) }\end{array}$ & $\begin{array}{l}\text { Sex }(\% \\
\text { female })\end{array}$ & Intervention & Control & $\begin{array}{l}\text { Primary; sec- } \\
\text { ondary out- } \\
\text { comes }\end{array}$ \\
\hline $\begin{array}{l}\text { e-BP } \\
2008[29]\end{array}$ & $\begin{array}{l}\text { 3-arm } \\
\text { RCT; } \\
\text { USA; } 12 \mathrm{~m}\end{array}$ & $\begin{array}{l}778 \text { people with Internet, } \\
\text { hypertension, from GPs: } \\
61.1 \% \text { obese }\end{array}$ & $59.1(8.5)$ & 52 & $\begin{array}{l}\text { (1) Website: BP self-monitor- } \\
\text { ing; }{ }^{b}(2) 1+\text { pharmacist sup- } \\
\text { port }^{c}\end{array}$ & $\begin{array}{l}\text { General website: } \\
\text { personal medical } \\
\text { record }\end{array}$ & $\begin{array}{l}\text { Change in dias- } \\
\text { tolic, systolic } \\
\text { and mean BP }\end{array}$ \\
\hline $\begin{array}{l}\text { Nolan } \\
2012[54]\end{array}$ & $\begin{array}{l}\text { 2-arm } \\
\text { RCT; } \\
\text { Canada; } 4 \\
\text { m }\end{array}$ & $\begin{array}{l}387 \text { people with hyperten- } \\
\text { sion via website: } 41 \% \\
\text { obese }\end{array}$ & $56.5(7.4)$ & 59 & $\begin{array}{l}\text { BP action plan website: assess- } \\
\text { ing motivational readiness, } \\
\text { advice, feedback, education }\end{array}$ & E-newsletters & $\begin{array}{l}\text { Change in dias- } \\
\text { tolic and sys- } \\
\text { tolic BP, and } \\
\text { pulse pressure }\end{array}$ \\
\hline $\begin{array}{l}\text { Bove } \\
2013[55]\end{array}$ & $\begin{array}{l}2 \text {-arm } \\
\text { RCT; } \\
\text { USA; } 6 \text { m }\end{array}$ & $\begin{array}{l}241 \text { people with elevated } \\
\text { BP from } 2 \text { clinics }\end{array}$ & $59.6(13.6)$ & 65 & $\begin{array}{l}\text { Website + telephone system: } \\
\text { education, self-monitoring } \\
\text { (BP, weight, exercise), online } \\
\text { nurse support, doctor in- } \\
\text { formed }\end{array}$ & $\begin{array}{l}\text { Provision of data } \\
\text { from initial assess- } \\
\text { ment, usual care }\end{array}$ & $\begin{array}{l}\text { Proportion of } \\
\text { participants } \\
\text { with controlled } \\
\text { BP at } 6 \mathrm{~m}\end{array}$ \\
\hline $\begin{array}{l}\text { Madsen } \\
2008[56]\end{array}$ & $\begin{array}{l}\text { 2-arm } \\
\text { RCT; Den- } \\
\text { mark; } 6 \mathrm{~m}\end{array}$ & $\begin{array}{l}236 \text { people with hyperten- } \\
\text { sion from } 10 \text { GPs }\end{array}$ & $55.9(11.7)$ & 50 & $\begin{array}{l}\text { Website: self-monitoring } \\
(\mathrm{BP}), \text { feedback from own } \\
\text { doctor by email }\end{array}$ & Usual care & $\begin{array}{l}\text { Change in ambu- } \\
\text { latory systolic } \\
\text { BP -at } 6 \mathrm{~m}\end{array}$ \\
\hline $\begin{array}{l}\text { Magid } \\
2013[57]\end{array}$ & $\begin{array}{l}\text { 2-arm } \\
\text { RCT; } \\
\text { USA; } 6 \mathrm{~m}\end{array}$ & $\begin{array}{l}348 \text { people with hyperten- } \\
\text { sion from } 10 \text { clinics }\end{array}$ & $60(11)$ & 40 & $\begin{array}{l}\text { Written educational material, } \\
\text { website: self-monitoring (BP), } \\
\text { pharmacist support, doctor } \\
\text { informed, reminders }\end{array}$ & $\begin{array}{l}\text { Written education } \\
\text { material, usual care }\end{array}$ & $\begin{array}{l}\text { Proportion of } \\
\text { participants } \\
\text { with controlled } \\
\text { BP at } 6 \mathrm{~m}\end{array}$ \\
\hline $\begin{array}{l}\text { McK- } \\
\text { instry } \\
2013[38]\end{array}$ & $\begin{array}{l}\text { 2-arm } \\
\text { RCT; Scot- } \\
\text { land; } 6 \mathrm{~m}\end{array}$ & $\begin{array}{l}401 \text { people with hyperten- } \\
\text { sion from } 20 \text { GPs }\end{array}$ & $60.7(11.2)$ & 40 & $\begin{array}{l}\text { Telemonitoring unit }+ \text { web- } \\
\text { site: self-monitoring (BP), } \\
\text { feedback from own doctor }\end{array}$ & Usual care & $\begin{array}{l}\text { Mean ambulato- } \\
\text { ry BP at } 6 \mathrm{~m}\end{array}$ \\
\hline $\begin{array}{l}\text { Thiboutot } \\
2013[58]\end{array}$ & $\begin{array}{l}\text { 2-arm clus- } \\
\text { ter RCT; } \\
\text { USA; } 12 \mathrm{~m}\end{array}$ & $\begin{array}{l}500 \text { patients with elevat- } \\
\text { ed BP from } 54 \text { GPs }\end{array}$ & $60.5(11.9)$ & 58 & $\begin{array}{l}\text { Website: self-monitoring (BP, } \\
\text { medication), feedback, re- } \\
\text { minders }\end{array}$ & $\begin{array}{l}\text { Different prevention } \\
\text { website (eg, breast } \\
\text { screening) }\end{array}$ & $\begin{array}{l}\text { BP control at } 12 \\
\mathrm{~m}\end{array}$ \\
\hline
\end{tabular}

\footnotetext{
abbreviations: BP: blood pressure; GP: general practitioner.

${ }^{\mathrm{b}}$ For studies with more than 2 arms, this arm was used for all analyses.

${ }^{\mathrm{c}}$ For studies with more than 2 arms, this arm was used for the subgroup analysis on blended interventions.
} 
Table 3. Characteristics of the studies included for the systematic review: interventions targeting weight loss and weight loss maintenance. ${ }^{\text {a }}$

\begin{tabular}{|c|c|c|c|c|c|c|c|}
\hline Study & $\begin{array}{l}\text { Setting } \\
\text { and study } \\
\text { length }\end{array}$ & Participants & $\begin{array}{l}\text { Age (years), } \\
\text { mean (SD) }\end{array}$ & $\begin{array}{l}\text { Sex }(\% \\
\text { female })\end{array}$ & Intervention & Control & $\begin{array}{l}\text { Primary; sec- } \\
\text { ondary out- } \\
\text { comes }\end{array}$ \\
\hline $\begin{array}{l}\text { Appel } 2011 \\
{[35]}\end{array}$ & $\begin{array}{l}\text { 3-arm } \\
\text { RCT; } \\
\text { USA; } 24 \\
\text { m }\end{array}$ & $\begin{array}{l}415 \text { people with obesi- } \\
\text { ty, } \geq 1 \mathrm{CV} \text { risk factor, } \\
\text { Internet from } 6 \text { primary } \\
\text { clinics }\end{array}$ & $54(10.2)$ & 64 & $\begin{array}{l}\text { (1) Website + mobile coach } \\
\text { support: education, self- } \\
\text { monitoring (weight, diet, } \\
\text { exercise), reminders, doctor } \\
\text { informed; }{ }^{\text {b,c }} \text { (2) } 1+\text { in-per- } \\
\text { son support }\end{array}$ & $\begin{array}{l}1 \text { (or } 2 \text { ) meetings } \\
\text { with coach; } \\
\text { brochure with } \\
\text { websites for weight } \\
\text { loss }\end{array}$ & $\begin{array}{l}\text { Change in } \\
\text { weight from } \\
\text { baseline to } 24 \\
\mathrm{~m}\end{array}$ \\
\hline $\begin{array}{l}\text { Bennett } \\
2012[28]\end{array}$ & $\begin{array}{l}\text { 2-arm } \\
\text { RCT; } \\
\text { USA; } 24 \\
\text { m }\end{array}$ & $\begin{array}{l}365 \text { obese people with } \\
\text { hypertension from } 3 \\
\text { clinics }\end{array}$ & $54.6(10.9)$ & 69 & $\begin{array}{l}\text { Website/interactive voice } \\
\text { response system: self-moni- } \\
\text { toring weight, setting, coach } \\
\text { support (phone), group ses- } \\
\text { sions, education }\end{array}$ & Self-help booklet & $\begin{array}{l}\text { Change in } \\
\text { weight at } 24 \mathrm{~m}\end{array}$ \\
\hline $\begin{array}{l}\text { Bennett } \\
2010 \text { [59] }\end{array}$ & $\begin{array}{l}\text { 2-arm } \\
\text { RCT; } \\
\text { USA; } 3 \\
\text { m }\end{array}$ & $\begin{array}{l}101 \text { obese people with } \\
\text { hypertension, Internet } \\
\text { from clinic }\end{array}$ & $54.4(8.1)$ & 48 & $\begin{array}{l}\text { Website: goal setting, self- } \\
\text { monitoring, behavioral skills } \\
\text { education, forum, coach } \\
\text { support (online, phone, face- } \\
\text { to-face) }\end{array}$ & $\begin{array}{l}\text { Folder on healthy } \\
\text { weight, usual care }\end{array}$ & $\begin{array}{l}\text { Change in } \\
\text { weight at } 12 \\
\text { weeks }\end{array}$ \\
\hline $\begin{array}{l}\text { Kraschnewsky } \\
2011[60]\end{array}$ & $\begin{array}{l}\text { 2-arm } \\
\text { RCT; } \\
\text { USA; } 3 \\
\text { m }\end{array}$ & $\begin{array}{l}100 \text { overweight people, } \\
\text { Internet via flyers/Inter- } \\
\text { net }\end{array}$ & $50.3(10.9)$ & 70 & $\begin{array}{l}\text { Website: target body weight, } \\
\text { monitoring, behavioral tips, } \\
\text { videos, weight loss plan, } \\
\text { tailored feedback, reminders }\end{array}$ & $\begin{array}{l}\text { Wait list, people } \\
\text { got access to web- } \\
\text { site after } 12 \text { weeks }\end{array}$ & Weight loss \\
\hline $\begin{array}{l}\text { Webber }^{\mathrm{d}} \\
2008[61]\end{array}$ & $\begin{array}{l}\text { 2-arm } \\
\text { RCT; } \\
\text { USA; } 4 \\
\text { m }\end{array}$ & $\begin{array}{l}66 \text { women, BMI } 25-40 \text {, } \\
\text { Internet from advertise- } \\
\text { ments }\end{array}$ & $50.0(9.9)$ & 100 & $\begin{array}{l}\text { Website: weight loss tips, } \\
\text { lessons, message board, self- } \\
\text { monitoring (weight, diet), } \\
\text { chat sessions }\end{array}$ & $\begin{array}{l}\text { All features of in- } \\
\text { tervention except } \\
\text { for online chat ses- } \\
\text { sions }\end{array}$ & $\begin{array}{l}\text { Not defined; } \\
\text { weight, BMI, } \\
\text { diet, exercise }\end{array}$ \\
\hline $\begin{array}{l}\text { POWER } \\
2014 \text { [62] }\end{array}$ & $\begin{array}{l}\text { 4-arm } \\
\text { RCT; } \\
\text { UK; } 12 \mathrm{~m}\end{array}$ & $\begin{array}{l}179 \text { people with BMI } \\
\geq 30 \mathrm{~kg} / \mathrm{m}^{2} \text { or } \geq 28 \\
\mathrm{~kg} / \mathrm{m}^{2}+\mathrm{CV} \text { risk factors } \\
\text { from } 5 \mathrm{GPs}\end{array}$ & $51.2(13.1)$ & 66 & $\begin{array}{l}\text { (1) Website: } 12 \text { self-manage- } \\
\text { ment sessions monitoring } \\
\text { (weight), nurse support } \\
\text { (email); }{ }^{\text {b,c }} \text { (2) } 1+3 \text { nurse } \\
\text { contacts; (3) } 1+7 \text { nurse } \\
\text { contacts }\end{array}$ & Usual care & Weight at $12 \mathrm{~m}$ \\
\hline \multicolumn{8}{|l|}{$\begin{array}{l}\text { Weight loss } \\
\text { maintenance }\end{array}$} \\
\hline $\begin{array}{l}\text { Stop Regain } \\
2008[41]\end{array}$ & $\begin{array}{l}\text { 3-arm } \\
\text { RCT; } \\
\text { USA; } 18 \\
\text { m }\end{array}$ & $\begin{array}{l}314 \text { people with } 10 \% \\
\text { weight loss in } 2 \text { years, } \\
\text { via advertisements }\end{array}$ & $51(10)$ & 81 & 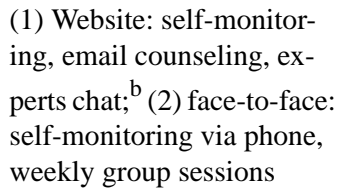 & (3) Newsletters & $\begin{array}{l}\text { Weight gain at } \\
18 \mathrm{~m}\end{array}$ \\
\hline $\begin{array}{l}\text { WLM } 2008 \\
\text { [40] }\end{array}$ & $\begin{array}{l}\text { 2-phase } \\
\text { 3-arm } \\
\text { RCT; } \\
\text { USA; } 30 \\
\text { m }\end{array}$ & $\begin{array}{l}1032 \text { people with } \geq 4 \mathrm{~kg} \\
\text { previous weight loss, } \\
\text { hypertension, Internet } \\
\text { via university/ medicare }\end{array}$ & $55.6(8.7)$ & 63 & $\begin{array}{l}\text { (1) Website: goal setting, } \\
\text { action plans, self-monitoring } \\
\text { (weight, PA, diet), educa- } \\
\text { tion, bulletin board, re- } \\
\text { minders, support } \\
\text { (email/phone); }{ }^{\text {b }} \text { (2) personal } \\
\text { contact (phone +face-to- } \\
\text { face) }\end{array}$ & $\begin{array}{l}\text { Printed lifestyle } \\
\text { guidelines, } 1 \text { visit } \\
\text { with coach }\end{array}$ & $\begin{array}{l}\text { Change in } \\
\text { weight }\end{array}$ \\
\hline
\end{tabular}

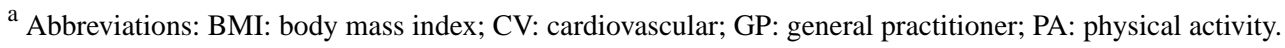

${ }^{\mathrm{b}}$ For studies with more than 2 arms, this arm was used for all analyses.

${ }^{\mathrm{c}}$ For studies with more than 2 arms, this arm was used for the subgroup analysis on blended interventions.
} 
${ }^{\mathrm{d}}$ Control arm consists of same interactive Internet platform as intervention arm.

Table 4. Characteristics of the studies included for the systematic review: interventions targeting physical activity and cholesterol. ${ }^{\text {a }}$

\begin{tabular}{|c|c|c|c|c|c|c|c|}
\hline Study & $\begin{array}{l}\text { Setting and } \\
\text { study } \\
\text { length }\end{array}$ & Participants & $\begin{array}{l}\text { Age (years), } \\
\text { mean (SD) }\end{array}$ & $\begin{array}{l}\text { Sex }(\% \\
\text { female })\end{array}$ & Intervention & Control & $\begin{array}{l}\text { Primary; sec- } \\
\text { ondary out- } \\
\text { comes }\end{array}$ \\
\hline \multicolumn{8}{|l|}{$\begin{array}{l}\text { Physical } \\
\text { activity }\end{array}$} \\
\hline $\begin{array}{l}\text { Richard- } \\
\text { son }^{\text {b }} 2010 \\
{[63]}\end{array}$ & $\begin{array}{l}\text { 2-arm } \\
\text { RCT; } \\
\text { USA; } 4 \text { m }\end{array}$ & $\begin{array}{l}324 \text { patients from clinic: } \\
12 \% \text { CHD, } 20 \% \text { DM } 2 \text {, } \\
62 \% \text { BMI }>30\end{array}$ & $52.0(11.4)$ & 65 & $\begin{array}{l}\text { Website as control + online } \\
\text { community forum }\end{array}$ & $\begin{array}{l}\text { Website: pedome- } \\
\text { ter, tailored feed- } \\
\text { back }\end{array}$ & $\begin{array}{l}\text { Change in aver- } \\
\text { age daily step } \\
\text { count, patient } \\
\text { attrition }\end{array}$ \\
\hline $\begin{array}{l}\text { Reid } \\
2011[30]\end{array}$ & $\begin{array}{l}\text { 2-arm } \\
\text { RCT; } \\
\text { Canada; } 12 \\
\text { m }\end{array}$ & $\begin{array}{l}223 \text { patients with a recent } \\
\text { CHD event, Internet via } \\
2 \text { cardiac centers }\end{array}$ & $56.4(9.0)$ & 16 & $\begin{array}{l}\text { Website: tutorials, exercise } \\
\text { plans, self-monitoring, spe- } \\
\text { cialist support }\end{array}$ & $\begin{array}{l}\text { Usual care, educa- } \\
\text { tion booklet }\end{array}$ & $\begin{array}{l}\text { Mean steps per } \\
\text { day }\end{array}$ \\
\hline $\begin{array}{l}\text { Ferney } \\
2009[64]\end{array}$ & $\begin{array}{l}\text { 2-arm } \\
\text { RCT; Aus- } \\
\text { tralia; } 6 \mathrm{~m}\end{array}$ & $\begin{array}{l}106 \text { inactive residents: } \\
58 \% \text { overweight }\end{array}$ & $52.0(4.6)$ & 72 & $\begin{array}{l}\text { Website: behavioral strate- } \\
\text { gies, goal setting, self-moni- } \\
\text { toring, advice, bulletin } \\
\text { board, news }\end{array}$ & $\begin{array}{l}\text { Website with mini- } \\
\text { mal interactivity }\end{array}$ & $\begin{array}{l}\text { Not defined; } \\
\text { physical activi- } \\
\text { ty, website use }\end{array}$ \\
\hline $\begin{array}{l}\text { Active af- } \\
\text { ter } 55 \\
2013[65]\end{array}$ & $\begin{array}{l}\text { 2-arm } \\
\text { RCT; } \\
\text { USA; } 3 \mathrm{~m}\end{array}$ & $\begin{array}{l}405 \text { sedentary people } \\
\text { with Internet via senior } \\
\text { centers/websites }\end{array}$ & $60.3(4.9)$ & 69 & $\begin{array}{l}\text { Website: education, goal } \\
\text { setting, exercise planning, } \\
11 \text { online exercise lessons, } \\
\text { self-monitoring, reminders }\end{array}$ & $\begin{array}{l}\text { No access to the } \\
\text { intervention }\end{array}$ & $\begin{array}{l}\text { Not defined; } \\
\text { physical activi- } \\
\text { ty, BMI }\end{array}$ \\
\hline $\begin{array}{l}\text { HEART } \\
2014[37]\end{array}$ & $\begin{array}{l}\text { 2-arm } \\
\text { RCT; New } \\
\text { Zealand; } 6 \\
\text { m }\end{array}$ & $\begin{array}{l}171 \text { people with stable } \\
\text { CHD, Internet from } 2 \\
\text { hospitals }\end{array}$ & $60.2(9.2)$ & 19 & $\begin{array}{l}\text { Exercise prescription, behav- } \\
\text { ioral strategies, Website: } \\
\text { videos, self-monitoring (ex- } \\
\text { ercise), education, reminders }\end{array}$ & Usual care & $\begin{array}{l}\text { Change in peak } \\
\text { oxygen uptake } \\
\text { from baseline to } \\
6 \mathrm{~m}\end{array}$ \\
\hline $\begin{array}{l}\text { Philips } \\
\text { Direct } \\
\text { Life } 2013 \\
{[66]}\end{array}$ & $\begin{array}{l}\text { 2-arm } \\
\text { RCT; } \\
\text { Nether- } \\
\text { lands; } 3 \mathrm{~m}\end{array}$ & $\begin{array}{l}235 \text { inactive people with } \\
\text { Internet through local } \\
\text { media }\end{array}$ & $64.8(2.9)$ & 41 & $\begin{array}{l}\text { Website: goal setting, self- } \\
\text { monitoring (exercise), e- } \\
\text { coach feedback }\end{array}$ & Waitlist control & $\begin{array}{l}\text { Change in phys- } \\
\text { ical activity }\end{array}$ \\
\hline $\begin{array}{l}\text { Suboc } \\
2014[67]\end{array}$ & $\begin{array}{l}\text { 3-arm } \\
\text { RCT; } \\
\text { USA; } 3 \text { m }\end{array}$ & $\begin{array}{l}114 \text { sedentary people } \\
\text { through media and Inter- } \\
\text { net }\end{array}$ & $63.0(7.0)$ & 34 & $\begin{array}{l}\text { (1) Pedometer; (2) website } \\
\text { + pedometer: exercise } \\
\text { strategies, goal setting, self- } \\
\text { monitoring (exercise) feed- } \\
\text { back, forum }\end{array}$ & No intervention & $\begin{array}{l}\text { Endothelial } \\
\text { function; vascu- } \\
\text { lar stiffness, } \\
\text { step count, exer- } \\
\text { cise }\end{array}$ \\
\hline $\begin{array}{l}\text { Peels } \\
2013[68]\end{array}$ & $\begin{array}{l}\text { 5-arm clus- } \\
\text { ter RCT; } \\
\text { Nether- } \\
\text { lands; } 12 \mathrm{~m}\end{array}$ & $\begin{array}{l}2140 \text { people from } 6 \mathrm{mu}- \\
\text { nicipal regions, } \pm 50 \% \\
\text { overweight }\end{array}$ & $63.2(8.4)$ & 51 & $\begin{array}{l}\text { (1) Printed feedback report; } \\
\text { (2) } 1+\text { local exercise tips; } \\
\text { (3) Web-based feedback re- } \\
\text { port; (4) } 3+\text { local exercise } \\
\text { tips }\end{array}$ & Waitlist control & $\begin{array}{l}\text { Physical activi- } \\
\text { ty }\end{array}$ \\
\hline \multicolumn{8}{|l|}{$\begin{array}{l}\text { Choles- } \\
\text { terol }\end{array}$} \\
\hline $\begin{array}{l}\text { Bloch }^{\text {b }} \\
2006[69]\end{array}$ & $\begin{array}{l}\text { 3-arm } \\
\text { RCT; } \\
\text { USA; } 6 \text { m }\end{array}$ & $\begin{array}{l}171 \text { employees with in- } \\
\text { creased cholesterol, DM } \\
\text { or CHD }\end{array}$ & $54.8(9.4)$ & - & $\begin{array}{l}\text { (1) Website + financial re- } \\
\text { ward; }(2) \text { website }+4 \text { class- } \\
\text { es, nurse support (phone) }\end{array}$ & $\begin{array}{l}\text { Website, } 10 \text {-year } \\
\text { CVD score, moni- } \\
\text { toring, goals, tai- } \\
\text { lored info }\end{array}$ & $\begin{array}{l}\text { LDL choles- } \\
\text { terol change at } \\
6 \mathrm{~m}\end{array}$ \\
\hline $\begin{array}{l}\text { Live well } \\
2013[70]\end{array}$ & $\begin{array}{l}\text { 2-arm } \\
\text { RCT; } \\
\text { USA; } 3 \text { m }\end{array}$ & $\begin{array}{l}61 \text { people with LDL } \\
\text { cholesterol } \geq 3.37 \\
\text { mmol/L, Internet from } \\
\text { primary clinics }\end{array}$ & $52.0(12.8)$ & 75 & $\begin{array}{l}\text { Web-based rate-your-plate } \\
\text { assessment, written educa- } \\
\text { tional material, Website: } \\
\text { goal setting, self-monitoring, } \\
\text { reminders }\end{array}$ & $\begin{array}{l}\text { Web-based rate- } \\
\text { your-plate assess- } \\
\text { ment }\end{array}$ & $\begin{array}{l}\text { Not defined; } \\
\text { cholesterol, } \\
\text { weight, Fram- } \\
\text { ingham risk } \\
\text { score }\end{array}$ \\
\hline
\end{tabular}

\footnotetext{
a Abbreviations: BMI: body mass index; CHD: coronary heart disease; CVD: cardiovascular disease; DM: diabetes mellitus; DM2: type 2 diabetes mellitus; LDL: low-density lipoprotein.

${ }^{\mathrm{b}}$ Control arm consists of same interactive Internet platform as intervention arm.

${ }^{\mathrm{c}}$ For studies with more than 2 arms, this arm was used for all analyses.
} 
Table 5. Characteristics of the studies included for the systematic review: interventions targeting multiple risk factors. ${ }^{\text {a }}$

\begin{tabular}{|c|c|c|c|c|c|c|c|}
\hline Study & $\begin{array}{l}\text { Setting and } \\
\text { study } \\
\text { length }\end{array}$ & Participants & $\begin{array}{l}\text { Age (years), } \\
\text { mean (SD) }\end{array}$ & $\begin{array}{l}\operatorname{Sex}(\% \\
\text { female })\end{array}$ & Intervention & Control & $\begin{array}{l}\text { Primary; sec- } \\
\text { ondary outcomes }\end{array}$ \\
\hline $\begin{array}{l}\text { Lindsay } \\
2008[71]\end{array}$ & $\begin{array}{l}\text { 2-arm } \\
\text { RCT; UK; } \\
6 \mathrm{~m}\end{array}$ & $\begin{array}{l}108 \text { heart patients living } \\
\text { in deprived areas }\end{array}$ & $62.9(6.0)$ & 33 & $\begin{array}{l}\text { eHealth portal: glossary, edu- } \\
\text { cation, local community links, } \\
\text { discussion forum }\end{array}$ & $\begin{array}{l}\text { No access to the } \\
\text { eHealth portal }\end{array}$ & $\begin{array}{l}\text { Not defined; be- } \\
\text { havior change } \\
\text { (exercise, smok- } \\
\text { ing, diet) }\end{array}$ \\
\hline $\begin{array}{l}\text { Heartcare } \\
\text { II } 2010 \\
{[72]}\end{array}$ & $\begin{array}{l}\text { 2-arm clus- } \\
\text { ter RCT; } \\
\text { USA; } 30 \mathrm{~m}\end{array}$ & $\begin{array}{l}282 \text { patients with chronic } \\
\text { heart disease needing } \\
\text { nursing care }\end{array}$ & $64.0(12.7)$ & 39 & $\begin{array}{l}\text { Personal health record: educa- } \\
\text { tion, monitoring, communica- } \\
\text { tion, goal setting, email, bul- } \\
\text { letin board }\end{array}$ & $\begin{array}{l}\text { Usual care as the } \\
\text { home care agencies } \\
\text { use to provide }\end{array}$ & $\begin{array}{l}\text { Satisfaction with } \\
\text { nursing care }\end{array}$ \\
\hline $\begin{array}{l}\text { Hughes } \\
2011[73]\end{array}$ & $\begin{array}{l}\text { 3-arm } \\
\text { RCT; } \\
\text { USA; } 12 \text { m }\end{array}$ & $\begin{array}{l}423 \text { senior university } \\
\text { employees with Internet, } \\
32 \% \text { overweight, } 46 \% \\
\text { obese }\end{array}$ & $51.0(7.0)$ & 82 & $\begin{array}{l}\text { (1) Coach for Web-based risk } \\
\text { assessment, lifestyle plan, } \\
\text { email, phone or in-person } \\
\text { contact; }^{c}(2) \text { website: risk } \\
\text { profile assessment, advice, } \\
\text { goal setting, action planning }\end{array}$ & $\begin{array}{l}\text { Printed list of } \\
\text { health promotion } \\
\text { programs }\end{array}$ & $\begin{array}{l}\text { Not defined; diet, } \\
\text { exercise, weight }\end{array}$ \\
\hline $\begin{array}{l}\text { Southard } \\
2003[26]\end{array}$ & $\begin{array}{l}\text { 2-arm } \\
\text { RCT; } \\
\text { USA; } 6 \mathrm{~m}\end{array}$ & $\begin{array}{l}104 \text { patients with CHD } \\
\text { or heart failure from } 10 \\
\text { hospitals, } 200 \mathrm{GPs} \text {, ad- } \\
\text { verts }\end{array}$ & $62.3(10.6)$ & 25 & $\begin{array}{l}\text { Website + nurse: education, } \\
\text { self-monitoring, discussion } \\
\text { group, links contact (email, } \\
\text { phone or mail), dietician }\end{array}$ & Usual care & $\begin{array}{l}\text { Not defined; } \\
\text { weight, exercise, } \\
\text { BP, lipid profile, } \\
\text { new CV events }\end{array}$ \\
\hline $\begin{array}{l}\text { Winett } \\
2007[74]\end{array}$ & $\begin{array}{l}\text { 3-arm clus- } \\
\text { ter RCT; } \\
\text { USA; } 16 \mathrm{~m}\end{array}$ & $\begin{array}{l}14 \text { churches with } 1071 \\
\text { members: } 57 \% \text { over- } \\
\text { weight, } 60 \% \text { sedentary }\end{array}$ & $51.4(15.7)$ & 67 & $\begin{array}{l}\text { (1) Website: education, goal } \\
\text { setting, pedometer; }^{\text {b }} \text { (2) } 1+ \\
\text { pulpit support }^{c}\end{array}$ & Waitlist condition & $\begin{array}{l}\text { Nutrition im- } \\
\text { provement, physi- } \\
\text { cal activity }\end{array}$ \\
\hline $\begin{array}{l}\text { Vernooij } \\
2012[27]\end{array}$ & $\begin{array}{l}\text { 2-arm } \\
\text { RCT; } \\
\text { Nether- } \\
\text { lands; } 12 \mathrm{~m}\end{array}$ & $\begin{array}{l}330 \text { patients with CVD, } \\
2 \text { risk factors, Internet via } \\
2 \text { hospitals }\end{array}$ & $59.9(8.4)$ & 25 & $\begin{array}{l}\text { Website: risk profile, self- } \\
\text { monitoring (BP, cholesterol), } \\
\text { treatment goal, nurse support, } \\
\text { news, medication changes }\end{array}$ & $\begin{array}{l}\text { Usual care by spe- } \\
\text { cialist or GP, re- } \\
\text { ceiving baseline } \\
\text { risk profile }\end{array}$ & $\begin{array}{l}\text { Relative change } \\
\text { in Framingham } \\
\text { heart risk score } \\
\text { after } 1 \text { year }\end{array}$ \\
\hline $\begin{array}{l}\text { Verheij- } \\
\text { den } 2004 \\
{[75]}\end{array}$ & $\begin{array}{l}\text { 2-arm } \\
\text { RCT; } \\
\text { Canada; } 8 \\
\text { m }\end{array}$ & $\begin{array}{l}146 \text { people with in- } \\
\text { creased CV risk, Internet } \\
\text { from } 14 \text { GPs }\end{array}$ & $63.0(10.5)$ & 45 & $\begin{array}{l}\text { Website: tailored information, } \\
\text { diet tool, bulletin board }\end{array}$ & Usual care & $\begin{array}{l}\text { Not defined; } \\
\text { BMI, BP, lipid } \\
\text { profile }\end{array}$ \\
\hline $\begin{array}{l}\text { Ross } \\
2004[33]\end{array}$ & $\begin{array}{l}\text { 2-arm } \\
\text { RCT; } \\
\text { USA; } 12 \mathrm{~m}\end{array}$ & $\begin{array}{l}107 \text { patients with heart } \\
\text { failure, Internet via clinic }\end{array}$ & $56.0(-)$ & 23 & $\begin{array}{l}\text { Online medical record (clini- } \\
\text { cal notes, laboratory reports, } \\
\text { test results), education, nurse } \\
\text { support }\end{array}$ & Usual care & $\begin{array}{l}\text { Change in self- } \\
\text { efficacy domain }\end{array}$ \\
\hline $\begin{array}{l}\text { Bove } \\
2011[76]\end{array}$ & $\begin{array}{l}\text { 2-arm } \\
\text { RCT; } \\
\text { USA; } 12 \mathrm{~m}\end{array}$ & $\begin{array}{l}465 \text { people with CVD } \\
\text { risk }>10 \% \text { via communi- } \\
\text { ty, clinics, churches }\end{array}$ & $61.0(10.0)$ & 46 & $\begin{array}{l}\text { Online telemedicine system: } \\
\text { laboratory and medication re- } \\
\text { view, self-monitoring (BP, } \\
\text { weight, pedometer), feedback, } \\
\text { education, own doctor in- } \\
\text { volved }\end{array}$ & $\begin{array}{l}\text { 4-months meetings } \\
\text { with nurse: review } \\
\text { data from logbooks }\end{array}$ & $\begin{array}{l}\text { Reduction in } \\
\text { Framingham 10- } \\
\text { year CVD risk } \\
\text { score }\end{array}$ \\
\hline $\begin{array}{l}\text { Keyser- } \\
\text { ling } 2014 \\
{[31]}\end{array}$ & $\begin{array}{l}\text { 2-arm } \\
\text { RCT; } \\
\text { USA; } 12 \mathrm{~m}\end{array}$ & $\begin{array}{l}385 \text { people with CHD } \\
\text { risk score } \geq 10 \% \text { but no } \\
\text { CVD from } 5 \text { GPs }\end{array}$ & $62.0(7.8)$ & 48 & $\begin{array}{l}\text { Website: CHD risk calculator, } \\
\text { advice, education, action } \\
\text { planning, goal setting. }\end{array}$ & $\begin{array}{l}\text { Same CHD risk } \\
\text { calculator, but in- } \\
\text { person and by } \\
\text { phone }\end{array}$ & $\begin{array}{l}\text { Framingham 10- } \\
\text { year CHD risk } \\
\text { score at } 4 \text { and } 12 \\
\mathrm{~m}\end{array}$ \\
\hline $\begin{array}{l}\text { Zullig } \\
2014[77]\end{array}$ & $\begin{array}{l}\text { 2-arm } \\
\text { RCT; } \\
\text { USA; } 3 \mathrm{~m}\end{array}$ & $\begin{array}{l}96 \text { people with CVD or } \\
\text { DM from primary clinics }\end{array}$ & $36.1(12.2)$ & 67 & $\begin{array}{l}\text { CVD risk assessment, web- } \\
\text { site: } 6 \text { modules with risk as- } \\
\text { sessments, goal setting, educa- } \\
\text { tion }\end{array}$ & $\begin{array}{l}\text { Printed informa- } \\
\text { tion on CVD }\end{array}$ & $\begin{array}{l}\text { Not defined; } \\
\text { Framingham 10- } \\
\text { year CVD risk } \\
\text { score, BMI, } \\
\text { smoking status, } \\
\text { systolic BP }\end{array}$ \\
\hline $\begin{array}{l}\text { Activate } \\
\text { your } \\
\text { Heart } \\
2014[78]\end{array}$ & $\begin{array}{l}\text { 2-arm } \\
\mathrm{RCT} ; \mathrm{UK} ; \\
6 \mathrm{~m}\end{array}$ & $\begin{array}{l}95 \text { people with stable } \\
\text { angina, Internet from } 9 \\
\text { GPs }\end{array}$ & $66.2(9.2)$ & 25 & $\begin{array}{l}\text { Website: CVD risk assess- } \\
\text { ment, education, goal setting, } \\
\text { self-monitoring, email/chat } \\
\text { with experts }\end{array}$ & Usual care with GP & $\begin{array}{l}\text { Change in step } \\
\text { count at } 6 \text { weeks } \\
\text { and } 6 \mathrm{~m}\end{array}$ \\
\hline
\end{tabular}




\begin{tabular}{|c|c|c|c|c|c|c|c|}
\hline Study & $\begin{array}{l}\text { Setting and } \\
\text { study } \\
\text { length }\end{array}$ & Participants & $\begin{array}{l}\text { Age (years), } \\
\text { mean (SD) }\end{array}$ & $\begin{array}{l}\text { Sex }(\% \\
\text { female })\end{array}$ & Intervention & Control & $\begin{array}{l}\text { Primary; sec- } \\
\text { ondary outcomes }\end{array}$ \\
\hline $\begin{array}{l}\text { e-Care } \\
2014 \text { [79] }\end{array}$ & $\begin{array}{l}2 \text {-arm RCT } \\
\text { USA } 6 \mathrm{~m}\end{array}$ & $\begin{array}{l}101 \text { people with BMI } \\
>26 \text {, elevated BP via } \\
\text { electronic health records }\end{array}$ & $56.9(7.0)$ & 42 & $\begin{array}{l}\text { Website + dietician: CVD risk } \\
\text { assessment, goal setting, ac- } \\
\text { tion planning, self-monitoring } \\
\text { (weight, BP, physical activity, } \\
\text { diet) }\end{array}$ & $\begin{array}{l}\text { Usual care, printed } \\
\text { report for patient } \\
\text { and doctor }\end{array}$ & $\begin{array}{l}\text { Change in sys- } \\
\text { tolic BP, weight } \\
\text { and } 10 \text {-year CVD } \\
\text { risk score }\end{array}$ \\
\hline $\begin{array}{l}\text { Greene } \\
2012[80]\end{array}$ & $\begin{array}{l}2 \text {-arm } \\
\text { RCT; } \\
\text { USA; } 6 \mathrm{~m}\end{array}$ & $\begin{array}{l}513 \text { employees }+ \text { families } \\
45 \% \text { overweight and } \\
48 \% \text { obese }\end{array}$ & $\begin{array}{l}60 \% \text { older } \\
\text { than } 50 \text { years }\end{array}$ & 79 & $\begin{array}{l}\text { Printed lifestyle guide, web- } \\
\text { site: online social network, } \\
\text { self-monitoring (weight, exer- } \\
\text { cise), goal setting, feedback }\end{array}$ & $\begin{array}{l}\text { Printed lifestyle } \\
\text { guide }\end{array}$ & $\begin{array}{l}\text { Not defined; } \\
\text { physical activity, } \\
\text { weight, lipid pro- } \\
\text { file }\end{array}$ \\
\hline $\begin{array}{l}\text { Holbrook } \\
2009[81]\end{array}$ & $\begin{array}{l}\text { 2-arm clus- } \\
\text { ter RCT; } \\
\text { Canada; } 12 \\
\text { m }\end{array}$ & $\begin{array}{l}46 \text { GPs with } 511 \text { people } \\
\text { with DM, } \geq 1 \mathrm{CV} \text { risk } \\
\text { factor }\end{array}$ & $60.7(12.5)$ & 49 & $\begin{array}{l}\text { Personal Web-based profile } \\
\text { overview for DM/CVRM } \\
\text { care, automated telephone re- } \\
\text { minders, summary for doctor, } \\
\text { doctor involved }\end{array}$ & Usual care & $\begin{array}{l}\text { Composite score } \\
\text { for process of } \\
\text { care }\end{array}$ \\
\hline $\begin{array}{l}\text { Diabetes } \\
\text { in Check } \\
2014 \text { [82] }\end{array}$ & $\begin{array}{l}2 \text {-arm } \\
\text { RCT; Aus- } \\
\text { tralia; } 9 \mathrm{~m}\end{array}$ & $\begin{array}{l}436 \text { people with DM, In- } \\
\text { ternet via DM network }\end{array}$ & $58.2(10.3)$ & 48 & $\begin{array}{l}\text { Website: self-monitoring (ex- } \\
\text { ercise) goal setting, education, } \\
\text { discussion board }\end{array}$ & $\begin{array}{l}\text { General website } \\
\text { with home page } \\
\text { and contacts page } \\
\text { only }\end{array}$ & $\begin{array}{l}\text { Not defined; } \\
\text { physical activity, } \\
\text { BMI }\end{array}$ \\
\hline
\end{tabular}

a Abbreviations: BMI: body mass index; BP: blood pressure; CHD: coronary heart disease; CV: cardiovascular; CVD: cardiovascular disease; CVRM: cardiovascular risk management; DM: diabetes mellitus; GP: general practitioner.

${ }^{\mathrm{b}}$ For studies with more than 2 arms, this arm was used for all analyses.

${ }^{\mathrm{c}}$ For studies with more than 2 arms, this arm was used for the subgroup analysis on blended interventions.

Figure 3. Effect on diastolic blood pressure (26 studies).

\section{Diastolic blood pressure $(\mathrm{mmHg})$}

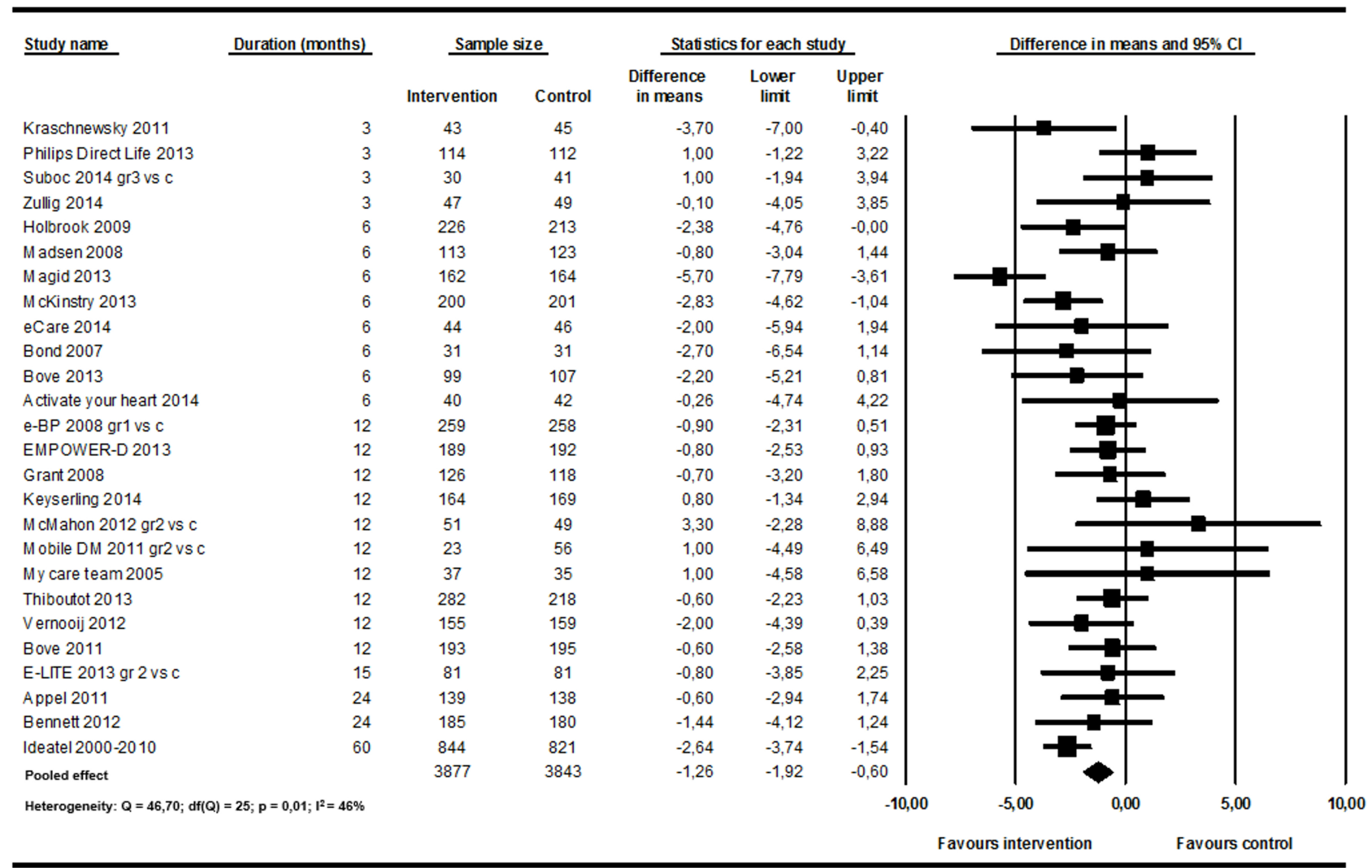

\section{Glycated Hemoglobin Alc}

A significant reduction in $\mathrm{HbA}_{1 \mathrm{c}}$ level favoring the intervention among patients with type 2 diabetes mellitus was found (21 studies; n=6518; Figure 4). The weighted mean difference for $\mathrm{HbA}_{1 \mathrm{c}}$ was $-0.13 \%$ (95\% CI -0.22 to $\left.-0.05 ; \mathrm{I}^{2}=74 \%\right)$. The jackknife procedure did not reveal one particular study responsible for high heterogeneity. 
Figure 4. Effect on glycated hemoglobin (21 studies).

\section{$\operatorname{HbA1c}(\%)$}

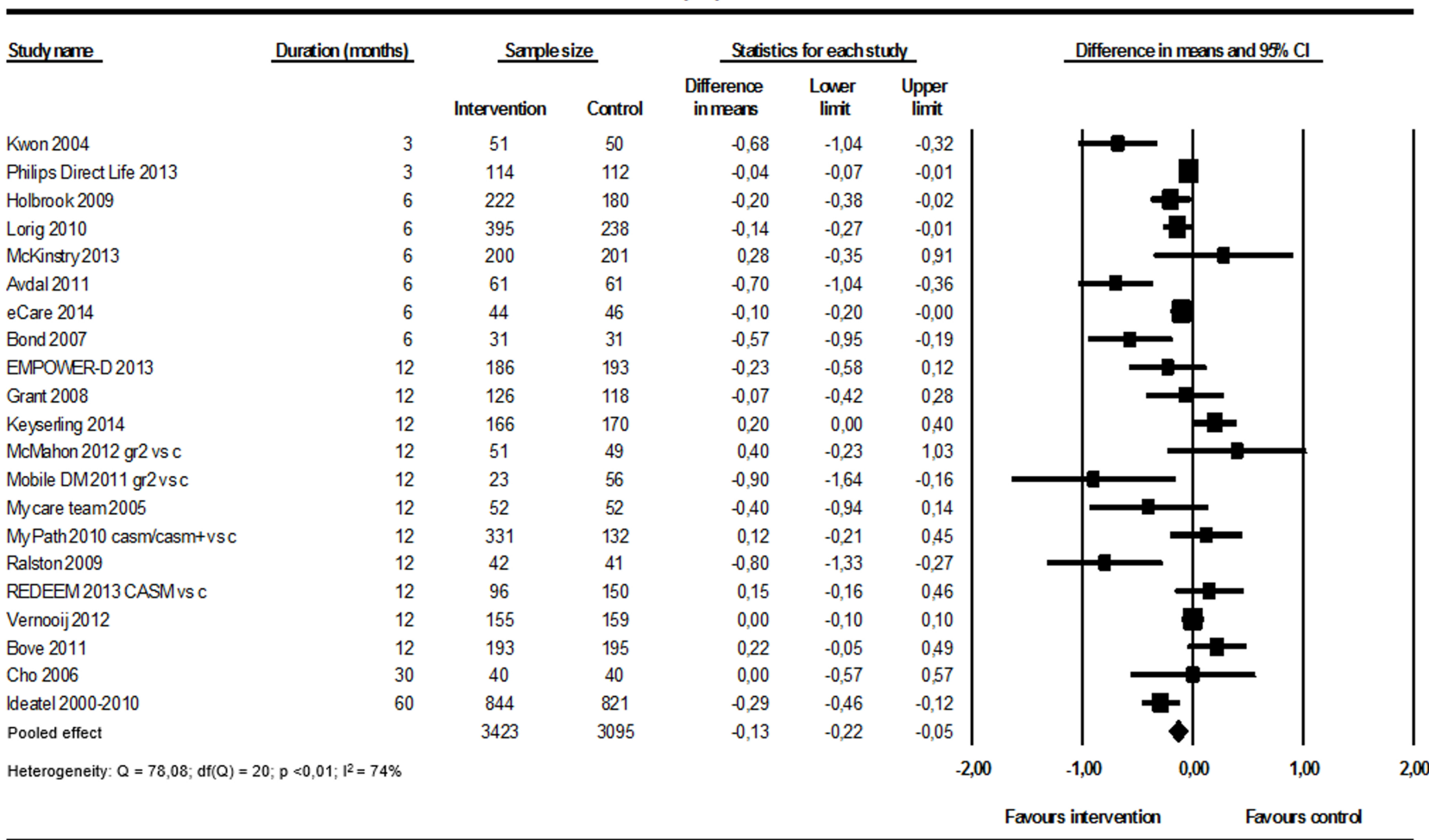

\section{Weight}

Fifteen studies tested interventions for weight loss and two studies tested interventions for maintenance of weight loss. The pooled analysis (17 studies; $n=3713$; Figure 5) showed a significant reduction in weight favoring the intervention (weighted mean difference $-1.34 \mathrm{~kg}, 95 \% \mathrm{CI}-1.91$ to -0.77 ; $\left.\mathrm{I}^{2}=61 \%\right)$. A sensitivity analysis leaving out the two studies on weight loss maintenance resulted in a similar effect size and level of heterogeneity. The jackknife procedure identified three studies contributing considerably to heterogeneity $[35,42,59]$.

Figure 5. Effect on weight (17 studies).

Weight (kg)

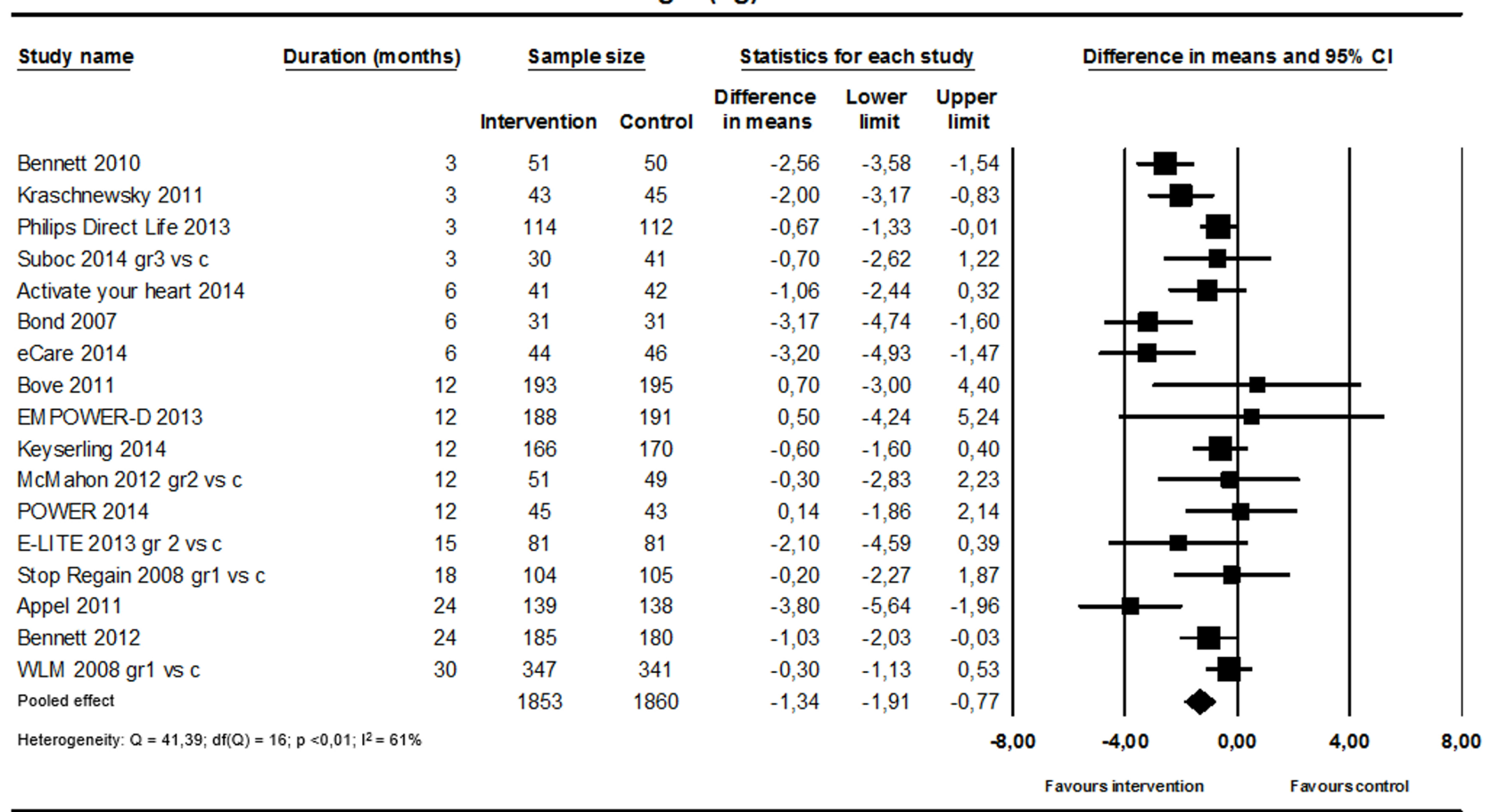




\section{Low-Density Lipoprotein Cholesterol}

A small but significant reduction in LDL cholesterol favoring the intervention was found (17 studies; $\mathrm{n}=5035$; Figure 6; weighted mean difference $-2.18 \mathrm{mg} / \mathrm{dL}, 95 \% \mathrm{CI}-3.96$ to -0.41 ; $\left.I^{2}=44 \%\right)$.

Figure 6. Effect on low-density lipoprotein cholesterol (17 studies).

\section{LDL-cholesterol (mg/dl)}

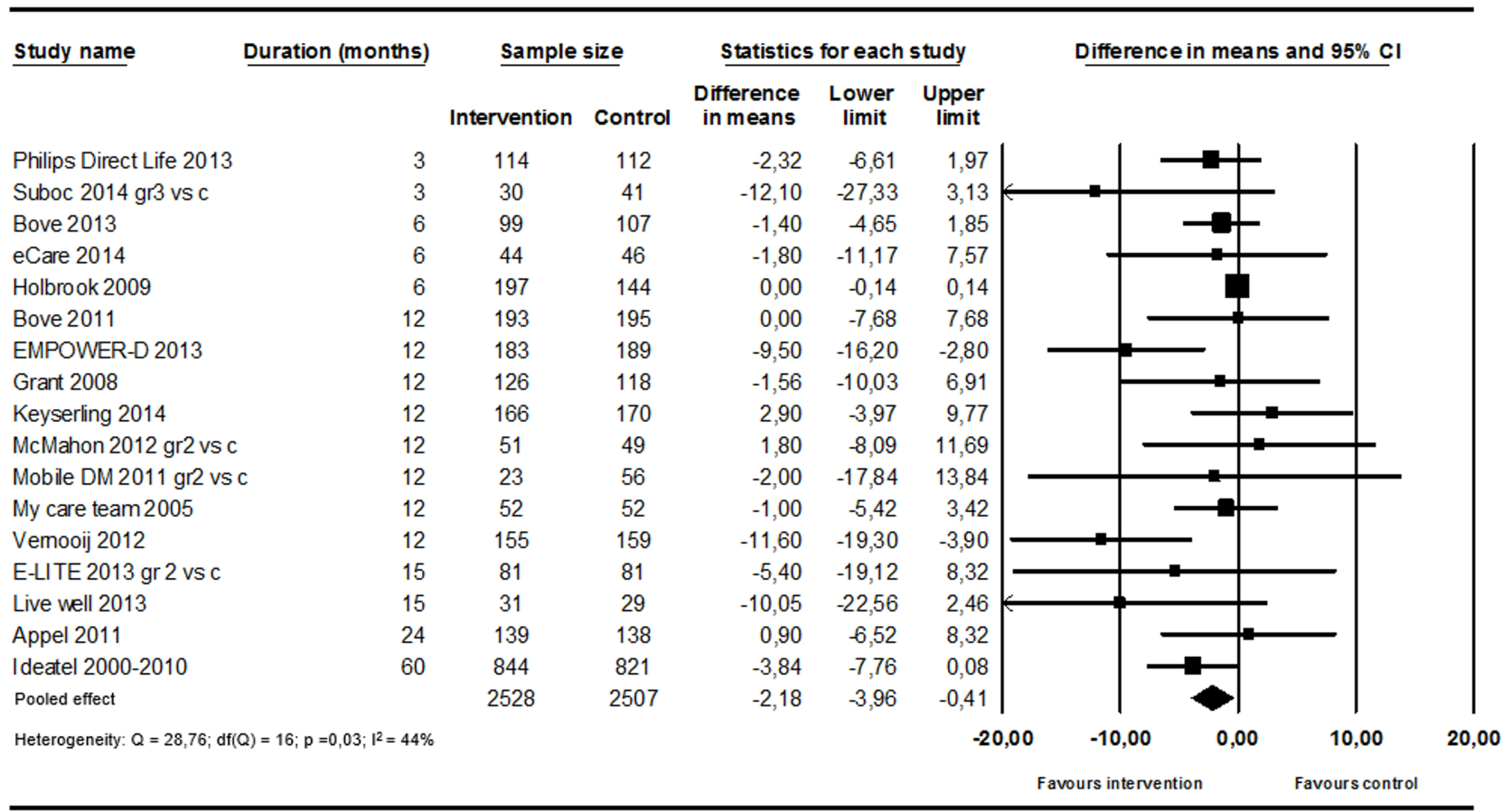

\section{Physical Activity}

Fourteen studies ( $\mathrm{n}=4444$; Figure 7) reported the effect on physical activity. Eight studies used self-reported physical activity levels in minutes per week, five studies used daily step counts obtained from pedometers, and one study measured physical activity with accelerometers. Because of the differences in measurement instruments, we calculated standardized mean differences. A small significant difference in increase of physical activity levels was found in favor of the intervention (weighted standardized mean difference $0.25,95 \%$ CI $0.10-0.39 ; \mathrm{I}^{2}=81 \%$ ), but heterogeneity was high. The jackknife procedure identified one study [65] driving a substantial part of heterogeneity; without this study, $\mathrm{I}^{2}$ was $68 \%$.

\section{Effect of Web-Based Interventions on Overall Cardiovascular Risk Profile, Cardiovascular Morbidity, and Mortality}

\section{Cardiovascular Composite Scores}

Nine studies ( $\mathrm{n}=2321$; Figure 8) reported a cardiovascular composite score. Five studies reported the Framingham 10-year cardiovascular disease risk score, three studies reported the Framingham 10-year coronary heart disease risk score, and one study reported a clinical composite score based on number of cardiovascular risk factors on target $\left(\mathrm{BP}, \mathrm{HbA}_{1 \mathrm{c}}\right.$, body mass index, LDL cholesterol, physical activity, albuminuria, foot ulcers, and smoking). Because of the differences between the composite scores, we calculated standardized mean differences. A small significant improvement of the cardiovascular composite scores was found (weighted standardized mean difference $-0.10,95 \%$ CI -0.18 to $-0.02 ; \mathrm{I}^{2}=0 \%$ ).

\section{General Effect of Web-Based Interventions on Cardiovascular Risk Factors}

Finally, we pooled the primary outcomes of the 37 studies $(n=11,021$; Figure 9) that defined a primary outcome (systolic BP: 7 studies; $\mathrm{HbA}_{1 \mathrm{c}}$ : 13 studies; weight: 8 studies; physical activity: 6 studies; cardiovascular composite score: 3 studies). The weighted standardized mean difference was -0.24 (95\% CI -0.31 to $-0.16 ; \mathrm{I}^{2}=69 \%$ ) in favor of the intervention. The jackknife procedure revealed that one study [57] somewhat influenced the heterogeneity; without this study, heterogeneity dropped to 64\%. The funnel plot (Multimedia Appendix 5) indicated that small studies reporting large effects might be overrepresented. The Egger's test confirmed that the funnel plot was not symmetrical $(P=.01)$. 
Figure 7. Effect on physical activity (14 studies).

\section{Physical activity (Hedges's g)}

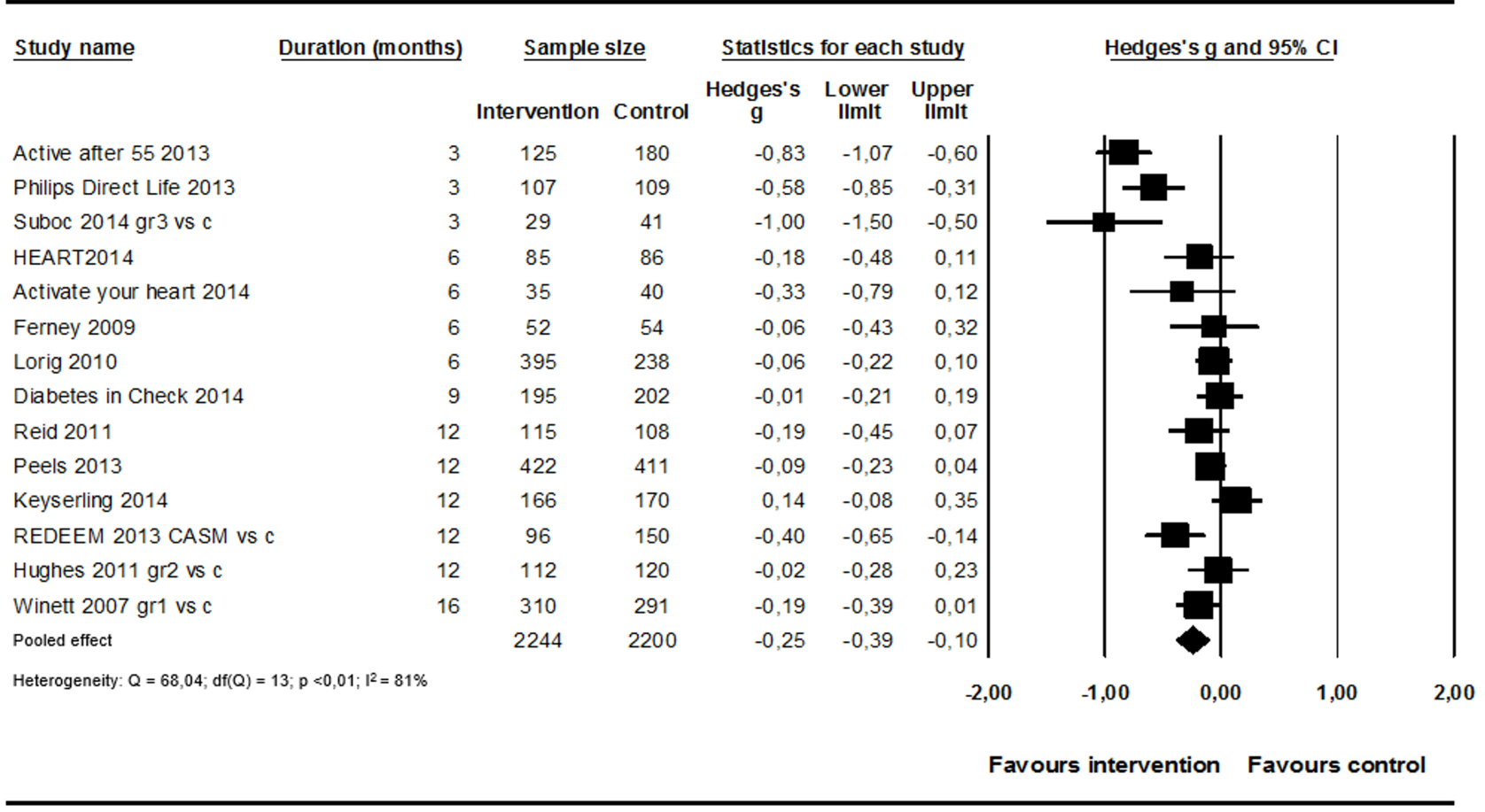

Figure 8. Effect on cardiovascular composite scores (9 studies).

Cardiovascular com posite scores (Hedges's g)

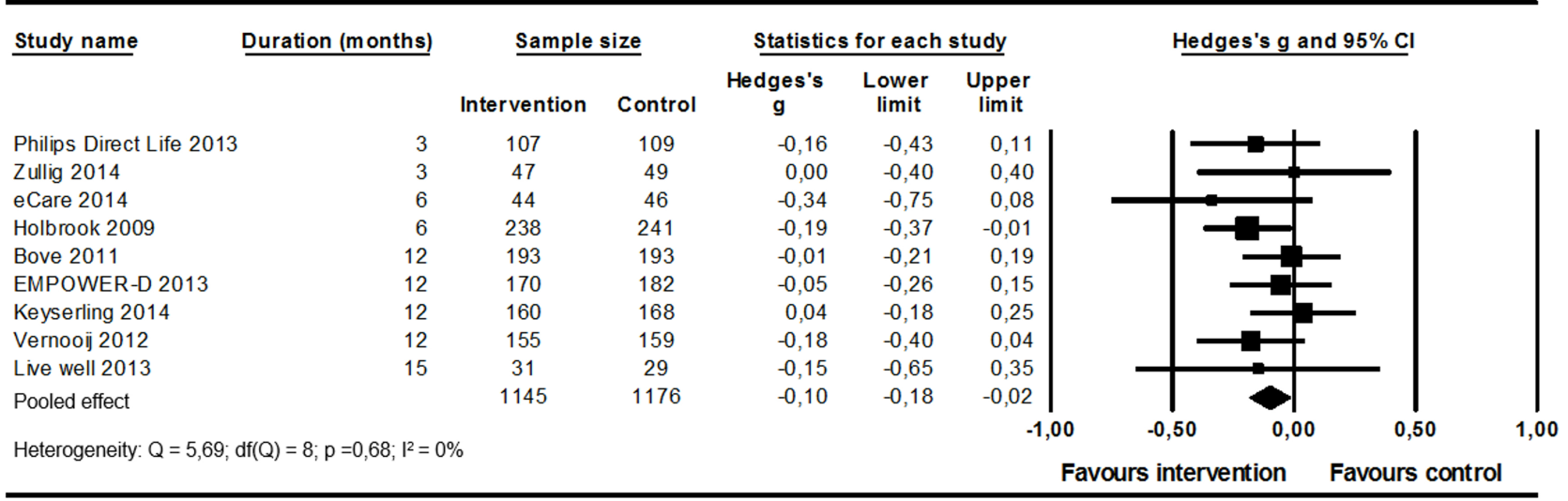


Figure 9. General effect on primary outcomes (37 studies).

Primary outcomes (Hedges's g)

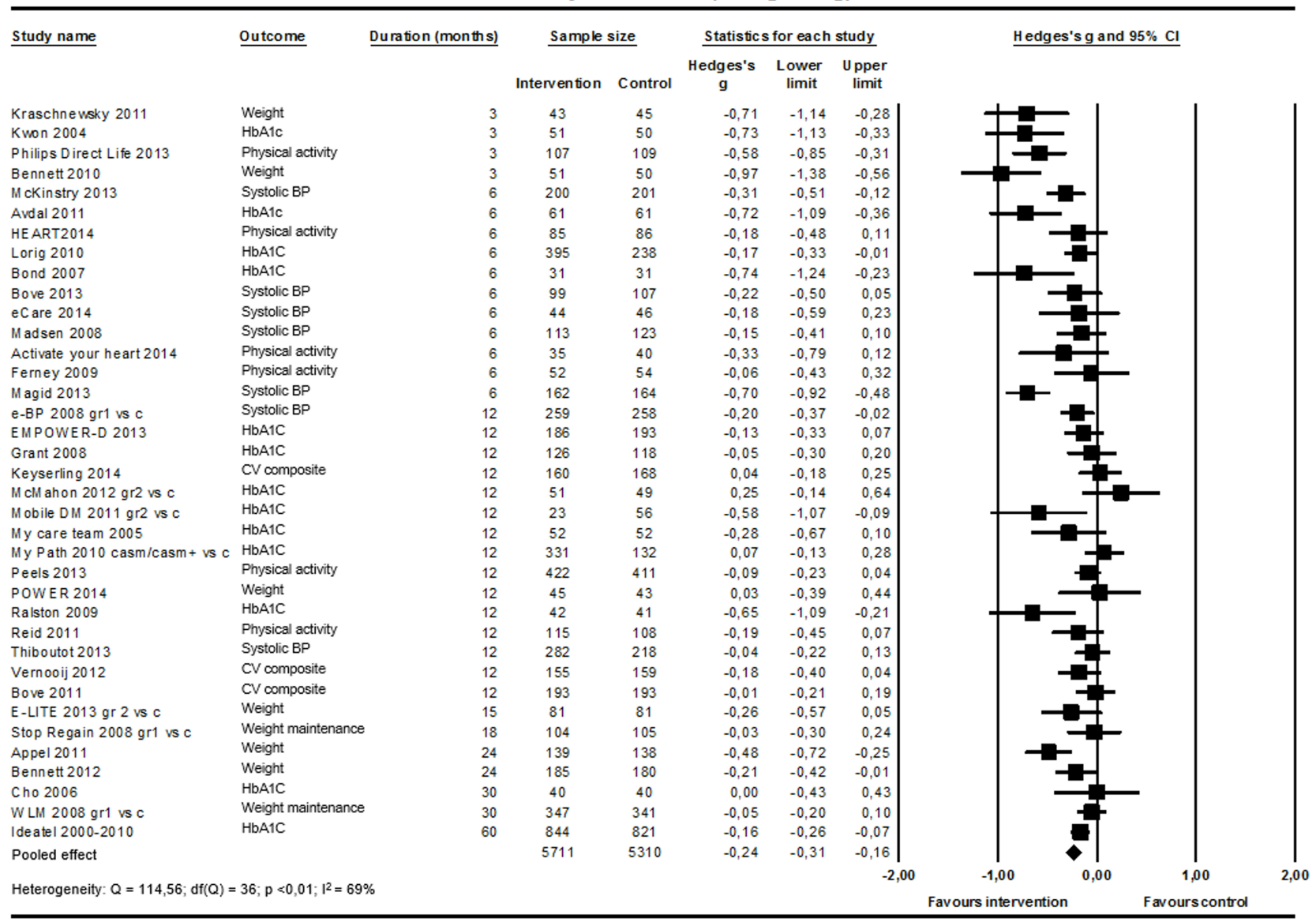

\section{Cardiovascular Morbidity and Total Mortality}

Six studies ( $\mathrm{n}=1904 ; 1$ short-term and 5 long-term studies) reported on cardiovascular event rates. The mean length of the studies was 13 months (range 6-24 months). The pooled analysis

Figure 10. Effect on cardiovascular event rates (6 studies). showed no difference in rate between groups (pooled OR 0.75 , 95\% CI 0.39-1.42; $\mathrm{I}^{2}=27 \%$; Figure 10). Total mortality rates were reported in 13 studies; in five studies, no deaths occurred and in the other eight studies, there were no differences between groups.

New cardiovascular events

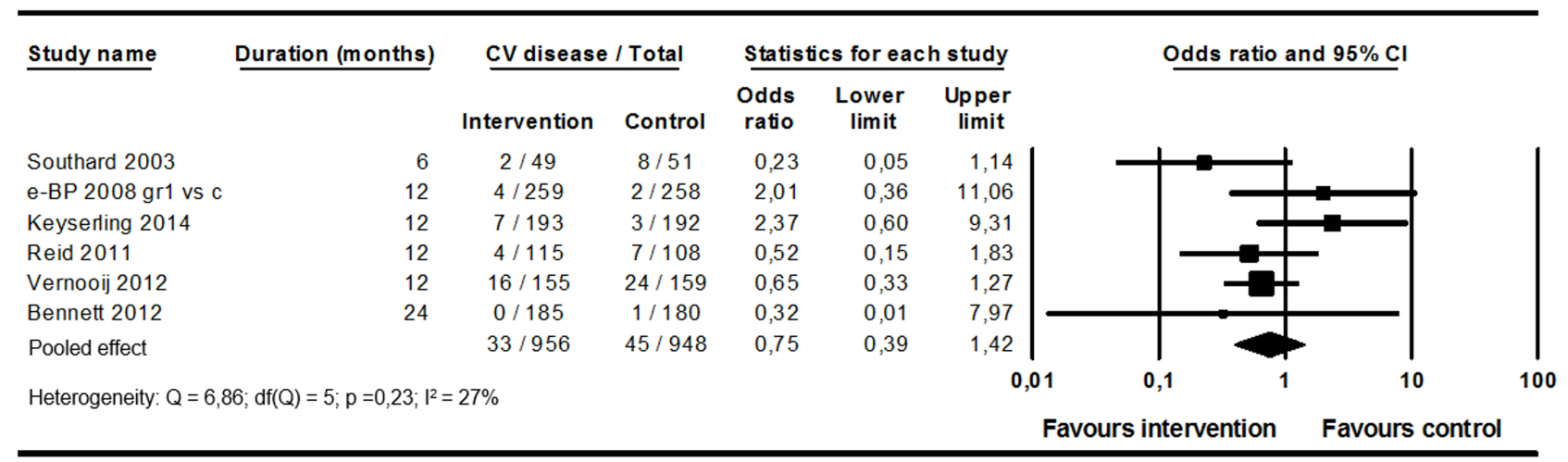


Figure 11. Association between study duration and effect size (Hedges' g). One outlier study (Ideatel) was removed from analysis.

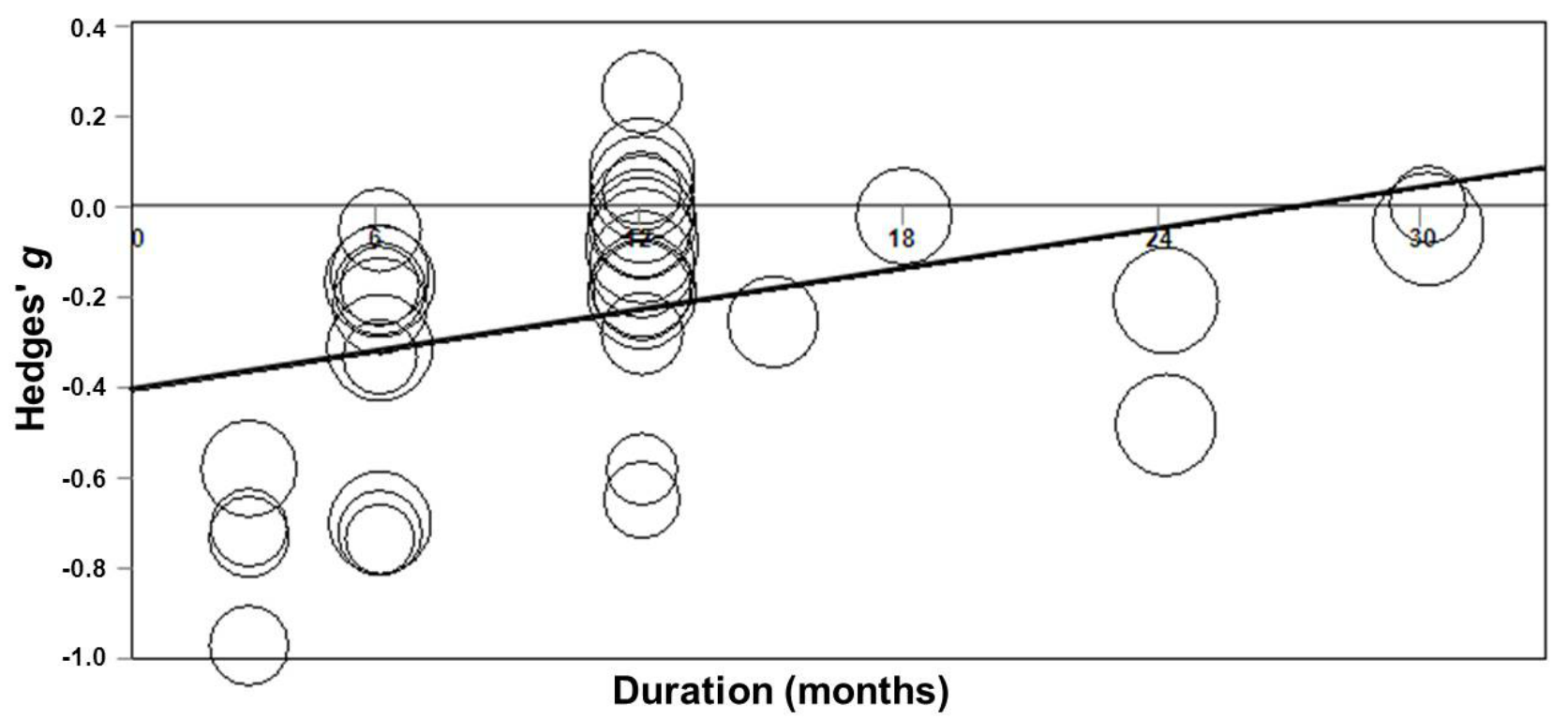

\section{Subgroup Analyses}

Results are summarized in Table 6. Within the analysis of pooled primary outcomes, the intervention effect was more pronounced in the short-term studies ( 15 studies; $\mathrm{n}=2934$; standardized mean difference $-0.43,95 \% \mathrm{CI}-0.57$ to $-0.29 ; \mathrm{I}^{2}=69 \%$ ) than in the long-term studies (22 studies; $\mathrm{n}=8087$; standardized mean difference $-0.12,95 \% \mathrm{CI}-0.19$ to $-0.06 ; \mathrm{I}^{2}=41 \%$ ). The same pattern was found for all other outcomes except for LDL cholesterol (Multimedia Appendix 6). There were no substantial differences in effect size between studies on primary prevention versus secondary prevention. To further explore the studies targeting primary prevention, we compared studies with populations of relatively low age (not all participants older than
50 years, $n=29$ ) with studies with populations of older age (all participants older than 50 years, $n=4$ ). The pooled effect size was larger for the studies with older participants (Hedges' $g=-0.30$ ) than for the studies with relatively younger participants (Hedges' $g=-0.23$ ), but the confidence intervals overlapped largely. We repeated the analysis of pooled primary outcomes on the sample of studies testing an Internet-only and a blended intervention. The intervention effect was more pronounced in the sample of blended studies ( 26 studies; $\mathrm{n}=7538$; standardized mean difference $-0.33,95 \%$ CI -0.43 to $-0.22 ; \mathrm{I}^{2}=78 \%$ ) compared to the sample of Internet-only studies (14 studies; $\mathrm{n}=4280$; standardized mean difference $-0.15,95 \% \mathrm{CI}-0.23$ to $-0.07 ; \mathrm{I}^{2}=40 \%$ ). 
Table 6. Subgroup analyses within the analysis of standardized primary outcomes.

\begin{tabular}{|c|c|c|c|c|}
\hline Subgroup & $\mathrm{N}$ of studies & Hedges' $g$ & $95 \% \mathrm{CI}$ & $\mathrm{I}^{2}$ \\
\hline \multicolumn{5}{|l|}{ Duration $^{\text {a }}$} \\
\hline Short ( $<12$ months $)$ & 15 & -0.43 & $-0.57,-0.29$ & $69 \%$ \\
\hline Long ( $\geq 12$ months) & 22 & -0.12 & $-0.19,-0.06$ & $41 \%$ \\
\hline \multicolumn{5}{|l|}{ Type of prevention ${ }^{\text {a }}$} \\
\hline Primary (including diabetes control) & 33 & -0.25 & $-0.32,-0.17$ & $72 \%$ \\
\hline Secondary & 4 & -0.20 & $-0.34,-0.06$ & $0 \%$ \\
\hline \multicolumn{5}{|l|}{ Primary prevention: age subgroups } \\
\hline Not all older than 50 years & 29 & -0.23 & $-0.33,-0.14$ & $72 \%$ \\
\hline All older than 50 years & 4 & -0.30 & $-0.51,-0.09$ & $80 \%$ \\
\hline Internet only vs control $^{\mathrm{c}}$ & 14 & -0.15 & $-0.23,-0.07$ & $40 \%$ \\
\hline Blended vs control $^{\mathrm{d}}$ & 26 & -0.33 & $-0.43,-0.22$ & $79 \%$ \\
\hline
\end{tabular}

${ }^{\text {a }}$ Subgroup analysis performed in the sample of studies that was used for the analysis of primary outcomes.

${ }^{\mathrm{b}}$ Subgroup analysis performed on the sample of studies that targeted primary prevention (including diabetes control).

${ }^{\mathrm{c}}$ Subgroup analysis performed on the sample of studies that evaluated an Internet-only intervention. In case a study tested multiple arms, the appropriate arm was included in the analysis.

${ }^{\mathrm{d}}$ Subgroup analysis performed on the sample of studies that evaluated a blended intervention. In case a study tested multiple arms, the appropriate arm was included in the analysis.

\section{Meta-Regression}

Because of the fairly consistent finding that treatment effects were higher in short-term studies than in long-term studies, we performed a mixed effects meta-regression to explore the association between study duration and effect size. The effect size seemed to become smaller in studies with longer follow-up, although the association was not significant (Hedges' $g=-0.321+0.006 *$ months; $P=.07)$. After removal of one outlier study [34] that had a very long follow-up (5 years), the effect size significantly decreased over time in studies lasting 3 to 32 months (Hedges' $g=-0.415+0.015 *$ months; $P=.008$; Figure. 11).

\section{Sensitivity Analyses for the Risk-of-Bias Assessment}

We performed sensitivity analyses for each of the six domains of bias assessed with the adapted Cochrane Risk of Bias Tool by comparing the standardized primary outcomes of the studies with the low risk and unknown/high risk of bias (Multimedia Appendix 7). There were no significant differences in pooled effect sizes in any of the domains except for the domain random sequence generation, in which the pooled effect was significantly larger in the subgroup of studies with unknown/high risk of bias.

\section{Discussion}

In this systematic review and meta-analysis, we found for people with elevated cardiovascular risk, Web-based interventions lead to improvement of systolic and diastolic $\mathrm{BP}, \mathrm{HbA}_{1 \mathrm{c}}$, weight, LDL cholesterol, physical activity levels, and cardiovascular risk composite scores. Only seven studies included participants all aged 50 years or older. Therefore, our conclusions apply for the population in middle age and beyond. Effects were more pronounced over the short term (study duration <12 months) and in studies that tested a blended intervention (combination of an Internet application and human support). We found no evidence for an effect on incident cardiovascular disease.

Our findings on single cardiovascular risk factors are consistent with conclusions of other meta-analyses in younger adult populations [19-21]. We found a significant reduction in systolic $\mathrm{BP}$ of $2.66 \mathrm{mmHg}$. A reduction of $3 \mathrm{mmHg}$ in systolic BP can lead to an $8 \%$ reduction in annual stroke mortality rate and a $5 \%$ reduction in annual coronary heart disease mortality rate [83]. We found a reduction of LDL cholesterol of $2.18 \mathrm{mg} / \mathrm{dL}$ (converted $=0.06 \mathrm{mmol} / \mathrm{L}$ ). A reduction of $0.5 \mathrm{mmol} / \mathrm{L}$ in $\mathrm{LDL}$ cholesterol for at least 2 years can lead to a reduction in coronary heart disease events of $20 \%$ [6]. Theoretically, assuming a linear relation, a reduction of $0.06 \mathrm{mmol} / \mathrm{L}$ could lead to a $2.4 \%$ reduction of coronary heart disease events. Thus, the effects on Internet interventions on BP reduction and, to a lesser extent, LDL cholesterol reduction, can be clinically relevant at the population level if reductions are maintained. In addition, we evaluated the effect on the complete cardiovascular risk profile and prevention of cardiovascular disease, which has not been performed before. One other systematic review without meta-analysis that evaluated Internet interventions for lifestyle change in older people reported that interventions with multiple components are more effective than interventions with a single component [84].

We found that the beneficial effects of Web-based interventions decline over time and effects are larger when interventions are combined with human support. Decreasing adherence over time was reported in several studies included in our meta-analysis and could be an important contributor to the decreasing effect over time. We were unable to formally test this because 
information on adherence and engagement was only reported by 22 studies and definitions varied widely. The identified effect moderators are not specific to Web-based interventions for cardiovascular risk factors $[85,86]$. Maintenance of behavioral change is notoriously complex and best achieved in longer studies with intensive interventions, more face-to-face, and more follow-up contacts. However, such interventions lead to high attrition rates, probably reflecting selection of the most motivated participants [87]. A careful balance should be sought between effectiveness and implementability when designing cardiovascular risk management interventions, whether or not an Internet-based approach is used.

Our results do not show a beneficial effect of Web-based interventions on incident cardiovascular disease. Although the declining effect over time could play a role, more likely explanations for these findings are the limited follow-up time of the studies to detect these outcomes (mean length of the studies was 13 months) and the fact that these outcomes were not the primary focus of these studies. Because of the latter, data collection may not have been systematic and adjudication of the data by an independent committee may be lacking. Therefore, we cannot draw strong conclusions from these findings.

The results of this study should be interpreted with caution because of several limitations. The methodological quality of the studies was fair, but none of the studies was double blind, rendering them prone to performance bias. Only 20 studies had a blinded outcome assessment, so detection bias may also be present. Because the sensitivity analyses for the risk-of-bias assessment did not reveal significant differences between the low risk and unknown/high risk-of-bias subgroups, except for the domain of random sequence generation, we think that our findings have not been largely affected by these potential sources of bias. Another limitation is the substantial heterogeneity in several of the meta-analyses that is, in part, explained by two effect modifiers: study duration and intervention type. Patient groups with a higher burden have a larger window of opportunity for improvement potentially resulting in larger intervention effects [88], which could also have contributed to heterogeneity. We could not draw firm conclusions on the difference between primary and secondary prevention, because only four studies on secondary prevention were included in this analysis. Last, there is a potential for publication bias and small study bias. Most of the studies with small sample sizes reported large effects and similar studies with null findings did not appear in the funnel plots (Multimedia Appendix 5).
Strengths of our study are the comprehensive search strategy, the quantitative meta-analysis, and the assessment of the effect of Web-based interventions for all cardiovascular risk factors using both intermediate and clinical outcomes. Our search strategy was comprehensive because we used a broad definition of Web-based interventions and only excluded telemedicine and mobile phone interventions. It was not always possible to set Web-based interventions apart from telemedicine and mobile phone interventions. As long as the Web-based program was the main component of the intervention, we judged the study eligible for our systematic review. By pooling the effect sizes on all different cardiovascular risk factors, we aimed to assess the overall effect of an Internet-based approach for people with increased risk of cardiovascular disease. This approach provides insight into the overall potential of Internet-based interventions in this field. Although basic computer literacy as an inclusion criterion probably led to selection of participants with a relatively high socioeconomic status, several studies included in the meta-analysis focused on people from medically underserved areas. Therefore, the external validity of the results might be acceptable and may be generalizable to middle-aged to older primary care populations with an increased risk of cardiovascular disease.

Our results show that Web-based interventions can be effective in improving the cardiovascular risk factor profile of middle-aged and older people, but effects are modest and can only have clinical relevance on the population level if sustained over time. Considering the current interest and focus on eHealth by policy makers, funding agencies, and a myriad of research and patient organizations $[89,90]$, it is important to evaluate the actual evidence base objectively. Unrealistic expectations of the effectiveness of Web-based interventions obscure the true challenges that have to be overcome first, including testing interventions that were designed specifically for older people, improving methodological robustness of studies, and improving sustainability of effects. On the macro level, trials can assess sustainability by prolonging follow-up, recording clinical events, and measuring surrogate cardiovascular outcomes (eg, BP, cholesterol levels, and weight) at multiple time points (eg, at 6, 12,24 , and 36 months). On the micro level, adherence should be evaluated by studying intervention usage through time with standardized evaluation methods. Sustainability is of particular importance because long-term effects are required for primary and secondary prevention to truly contribute to the prevention of cardiovascular disease. Web-based interventions combined with human support are more promising than Internet-only interventions.

\section{Acknowledgments}

We thank R Spijker, medical librarian at the Academic Medical Centre of Amsterdam, for his assistance in designing and conducting the search and M Siervo, lecturer in Nutrition and Ageing at the Institute for Ageing and Health, Newcastle University, for his advice on our statistical analysis plan. We also thank the study authors (LA Volk, CC Quinn, and JD Ralston) who provided additional data. The research leading to these results has received funding from the European Union Seventh Framework Programme (FP7/2007-2013) under grant agreement no. 305654. The funders had no role in study design, data collection and analysis, decision to publish, or preparation of the manuscript. 


\section{Authors' Contributions}

CRB had full access to all data in the study and takes responsibility for the integrity of the data and the accuracy of the data analysis. ER, EMvC, WvG, CB, MK, SA, and HS conceived the study. CRB, BS, and ER wrote the study protocol and analysis plan. CRB and BS collected and extracted the data, with support from ER and EMvC. CRB performed the statistical analysis, supported by BS, ER, WB, and WvG. CRB and ER drafted the manuscript and all authors critically revised the manuscript for important intellectual content.

\section{Conflicts of Interest}

None declared.

\section{Multimedia Appendix 1}

Research protocol.

[PDF File (Adobe PDF File), 63KB-Multimedia Appendix 1]

\section{Multimedia Appendix 2}

Comprehensive search strategy.

[PDF File (Adobe PDF File), 31KB-Multimedia Appendix 2]

\section{Multimedia Appendix 3}

Data-extraction form.

[PDF File (Adobe PDF File), 37KB-Multimedia Appendix 3]

\section{Multimedia Appendix 4}

Summary of the risk of bias assessment.

[PDF File (Adobe PDF File), 296KB-Multimedia Appendix 4]

\section{Multimedia Appendix 5}

Funnel plots.

[PDF File (Adobe PDF File), 77KB-Multimedia Appendix 5]

\section{Multimedia Appendix 6}

Subgroup analysis: study-duration.

[PDF File (Adobe PDF File), 55KB-Multimedia Appendix 6]

\section{Multimedia Appendix 7}

Risk of bias assessment: sensitivity analyses for the domains of risk of bias.

[PDF File (Adobe PDF File), 35KB-Multimedia Appendix 7]

\section{References}

1. Meier CA, Fitzgerald MC, Smith JM. eHealth: extending, enhancing, and evolving health care. Annu Rev Biomed Eng 2013;15:359-382. [doi: 10.1146/annurev-bioeng-071812-152350] [Medline: 23683088]

2. World Health Organization, National Institute on Aging, National Institutes of Health. Global Health and Aging. Geneva: World Health Organization; 2011. URL: http://www.who.int/ageing/publications/global health.pdf [accessed 2016-02-22] [WebCite Cache ID 6fU0boctf]

3. [No authors listed]. An epidemic of risk factors for cardiovascular disease. Lancet 2011 Feb 12;377(9765):527. [doi: 10.1016/S0140-6736(11)60182-7] [Medline: 21315931]

4. Chow CK, Jolly S, Rao-Melacini P, Fox KA, Anand SS, Yusuf S. Association of diet, exercise, and smoking modification with risk of early cardiovascular events after acute coronary syndromes. Circulation 2010 Feb 16;121(6):750-758 [FREE Full text] [doi: 10.1161/CIRCULATIONAHA.109.891523] [Medline: 20124123] 
5. Law MR, Morris JK, Wald NJ. Use of blood pressure lowering drugs in the prevention of cardiovascular disease: meta-analysis of 147 randomised trials in the context of expectations from prospective epidemiological studies. BMJ 2009;338:b1665 [FREE Full text] [Medline: 19454737]

6. Law MR, Wald NJ, Rudnicka AR. Quantifying effect of statins on low density lipoprotein cholesterol, ischaemic heart disease, and stroke: systematic review and meta-analysis. BMJ 2003 Jun 28;326(7404):1423 [FREE Full text] [doi: 10.1136/bmj.326.7404.1423] [Medline: 12829554]

7. Kotseva K, Wood D, De Backer G, De Bacquer D, Pyörälä K, Keil U. EUROASPIRE III: a survey on the lifestyle, risk factors and use of cardioprotective drug therapies in coronary patients from 22 European countries. Eur J Cardiovasc Prev Rehabil 2009 Apr;16(2):121-137. [doi: 10.1097/HJR.0b013e3283294b1d] [Medline: 19287307]

8. Nieuwlaat R, Schwalm J, Khatib R, Yusuf S. Why are we failing to implement effective therapies in cardiovascular disease? Eur Heart J 2013 May;34(17):1262-1269 [FREE Full text] [doi: 10.1093/eurheartj/ehs481] [Medline: 23376448]

9. Al Hamid A, Ghaleb M, Aljadhey H, Aslanpour Z. A systematic review of qualitative research on the contributory factors leading to medicine-related problems from the perspectives of adult patients with cardiovascular diseases and diabetes mellitus. BMJ Open 2014;4(9):e005992 [FREE Full text] [doi: 10.1136/bmjopen-2014-005992] [Medline: 25239295]

10. Sabaté E. Adherence to Long-Term Therapies: Evidence for Action. Geneva: World Health Organization; 2003.

11. Griffiths F, Lindenmeyer A, Powell J, Lowe P, Thorogood M. Why are health care interventions delivered over the internet? A systematic review of the published literature. J Med Internet Res 2006;8(2):e10 [FREE Full text] [doi: 10.2196/jmir.8.2.e10] [Medline: 16867965]

12. McManus RJ, Mant J, Bray EP, Holder R, Jones MI, Greenfield S, et al. Telemonitoring and self-management in the control of hypertension (TASMINH2): a randomised controlled trial. Lancet 2010 Jul 17;376(9736):163-172. [doi: 10.1016/S0140-6736(10)60964-6] [Medline: 20619448]

13. Briasoulis A, Agarwal V, Tousoulis D, Stefanadis C. Effects of antihypertensive treatment in patients over 65 years of age: a meta-analysis of randomised controlled studies. Heart 2014 Feb;100(4):317-323. [doi: 10.1136/heartjnl-2013-304111] [Medline: 23813846]

14. Beckett NS, Peters R, Fletcher AE, Staessen JA, Liu L, Dumitrascu D, et al. Treatment of hypertension in patients 80 years of age or older. N Engl J Med 2008 May 1;358(18):1887-1898. [doi: 10.1056/NEJMoa0801369] [Medline: 18378519]

15. Peters R, Beckett N, Forette F, Tuomilehto J, Clarke R, Ritchie C, et al. Incident dementia and blood pressure lowering in the Hypertension in the Very Elderly Trial cognitive function assessment (HYVET-COG): a double-blind, placebo controlled trial. Lancet Neurol 2008 Aug;7(8):683-689 [FREE Full text] [doi: 10.1016/S1474-4422(08)70143-1] [Medline: 18614402]

16. Ritchie K, Carrière I, Ritchie CW, Berr C, Artero S, Ancelin M. Designing prevention programmes to reduce incidence of dementia: prospective cohort study of modifiable risk factors. BMJ 2010;341:c3885 [FREE Full text] [Medline: 20688841]

17. Seybert H. Eurostat: Industry, Trade and Services. 2012 Dec 13. Internet use in households and by individuals in 2012 URL: http://ec.europa.eu/eurostat/documents/3433488/5585460/KS-SF-12-050-EN.PDF/ 39000dab-e2b7-49b2-bc4b-6aad0bf01279 [accessed 2016-02-22] [WebCite Cache ID 6fU2pZrEB]

18. Civljak M, Stead LF, Hartmann-Boyce J, Sheikh A, Car J. Internet-based interventions for smoking cessation. Cochrane Database Syst Rev 2013;7:CD007078. [doi: 10.1002/14651858.CD007078.pub4] [Medline: 23839868]

19. Liu S, Dunford SD, Leung YW, Brooks D, Thomas SG, Eysenbach G, et al. Reducing blood pressure with Internet-based interventions: a meta-analysis. Can J Cardiol 2013 May;29(5):613-621. [doi: 10.1016/j.cjca.2013.02.007] [Medline: 23618507]

20. Wieland LS, Falzon L, Sciamanna CN, Trudeau KJ, Brodney S, Schwartz JE, et al. Interactive computer-based interventions for weight loss or weight maintenance in overweight or obese people. Cochrane Database Syst Rev 2012;8:CD007675 [FREE Full text] [doi: 10.1002/14651858.CD007675.pub2] [Medline: 22895964]

21. Pal K, Eastwood SV, Michie S, Farmer AJ, Barnard ML, Peacock R, et al. Computer-based diabetes self-management interventions for adults with type 2 diabetes mellitus. Cochrane Database Syst Rev 2013;3:CD008776. [doi: 10.1002/14651858.CD008776.pub2] [Medline: 23543567]

22. Fifth Joint Task Force of the European Society of Cardiology, European Association of Echocardiography, European Association of Percutaneous Cardiovascular Interventions, European Heart Rhythm Association, Heart Failure Association, European Association for Cardiovascular Prevention \& Rehabilitation, European Atherosclerosis Society, International Society of Behavioural Medicine, European Stroke Organisation, European Society of Hypertension, European Association for the Study of Diabetes, European Society of General Practice/Family Medicine, International Diabetes Federation Europe, European Heart Network. European Guidelines on cardiovascular disease prevention in clinical practice (version 2012): the Fifth Joint Task Force of the European Society of Cardiology and Other Societies on Cardiovascular Disease Prevention in Clinical Practice (constituted by representatives of nine societies and by invited experts). Eur J Prev Cardiol 2012 Aug;19(4):585-667. [doi: 10.1177/2047487312450228] [Medline: 22763626]

23. Pearson TA, Palaniappan LP, Artinian NT, Carnethon MR, Criqui MH, Daniels SR, American Heart Association Council on Epidemiology and Prevention. American Heart Association Guide for Improving Cardiovascular Health at the Community Level, 2013 update: a scientific statement for public health practitioners, healthcare providers, and health policy makers. Circulation 2013 Apr 23;127(16):1730-1753 [FREE Full text] [doi: 10.1161/CIR.0b013e31828f8a94] [Medline: 23519758] 
24. Ritterband LM, Thorndike F. Internet interventions or patient education web sites? J Med Internet Res 2006;8(3):e18; author reply e19 [FREE Full text] [doi: 10.2196/jmir.8.3.e18] [Medline: 17032634]

25. Bennett GG, Glasgow RE. The delivery of public health interventions via the Internet: actualizing their potential. Annu Rev Public Health 2009;30:273-292. [doi: 10.1146/annurev.publhealth.031308.100235] [Medline: 19296777]

26. Southard B, Southard D, Nuckolls J. Clinical trial of an internet-based case management system for secondary prevention of heart disease. J Cardiopulm Rehabi 2003;23(5):341-348. [Medline: 14512778]

27. Vernooij W, Kaasjager H, Van Der Graaf Y, Wierdsma J, Grandjean H, Hovens M. Internet based vascular risk factor management for patients with clinically manifest vascular disease: randomised controlled trial. BMJ (Online) 2012;344(7863):e3750. [Medline: 22692651]

28. Bennett G, Warner E, Glasgow R, Askew S, Goldman J, Ritzwoller D, Be Fit, Be Well Study Investigators. Obesity treatment for socioeconomically disadvantaged patients in primary care practice. Arch Intern Med 2012 Apr 9;172(7):565-574 [FREE Full text] [doi: 10.1001/archinternmed.2012.1] [Medline: 22412073]

29. Green B, Cook A, Ralston J, Fishman P, Catz S, Carlson J, et al. Effectiveness of home blood pressure monitoring, Web communication, and pharmacist care on hypertension control: a randomized controlled trial. JAMA 2008 Jun 25;299(24):2857-2867 [FREE Full text] [doi: 10.1001/jama.299.24.2857] [Medline: 18577730]

30. Reid R, Morrin L, Beaton L, Papadakis S, Kocourek J, McDonnell L, et al. Randomized trial of an internet-based computer-tailored expert system for physical activity in patients with heart disease. Eur J Prev Cardiol 2012 Dec;19(6):1357-1364. [doi: 10.1177/1741826711422988] [Medline: 21903744]

31. Keyserling TC, Sheridan SL, Draeger LB, Finkelstein EA, Gizlice Z, Kruger E, et al. A comparison of live counseling with a web-based lifestyle and medication intervention to reduce coronary heart disease risk: a randomized clinical trial. JAMA Intern Med 2014 Jul;174(7):1144-1157 [FREE Full text] [doi: 10.1001/jamainternmed.2014.1984] [Medline: 24861959]

32. Quinn C, Shardell M, Terrin M, Barr E, Ballew S, Gruber-Baldini A. Cluster-randomized trial of a mobile phone personalized behavioral intervention for blood glucose control. Diabetes Care 2011 Sep;34(9):1934-1942 [FREE Full text] [doi: 10.2337/dc11-0366] [Medline: 21788632]

33. Ross S, Moore L, Earnest M, Wittevrongel L, Lin C. Providing a web-based online medical record with electronic communication capabilities to patients with congestive heart failure: randomized trial. J Med Internet Res 2004 May 14;6(2):e12 [FREE Full text] [doi: 10.2196/jmir.6.2.e12] [Medline: 15249261]

34. Shea S, Weinstock R, Teresi J, Palmas W, Starren J, Cimino J, IDEATel Consortium. A randomized trial comparing telemedicine case management with usual care in older, ethnically diverse, medically underserved patients with diabetes mellitus: 5 year results of the IDEATel study. J Am Med Inform Assoc 2009;16(4):446-456 [FREE Full text] [doi: 10.1197/jamia.M3157] [Medline: 19390093]

35. Appel L, Clark J, Yeh H, Wang N, Coughlin J, Daumit G, et al. Comparative effectiveness of weight-loss interventions in clinical practice. N Engl J Med 2011 Nov 24;365(21):1959-1968 [FREE Full text] [doi: 10.1056/NEJMoa1108660] [Medline: 22085317]

36. Ma J, Yank V, Xiao L, Lavori PW, Wilson SR, Rosas LG, et al. Translating the Diabetes Prevention Program lifestyle intervention for weight loss into primary care: a randomized trial. JAMA Intern Med 2013 Jan 28;173(2):113-121 [FREE Full text] [doi: 10.1001/2013.jamainternmed.987] [Medline: 23229846]

37. Maddison R, Pfaeffli L, Whittaker R, Stewart R, Kerr A, Jiang Y, et al. A mobile phone intervention increases physical activity in people with cardiovascular disease: Results from the HEART randomized controlled trial. Eur J Prev Cardiol 2015 Jun;22(6):701-709. [doi: 10.1177/2047487314535076] [Medline: 24817694]

38. McKinstry B, Hanley J, Wild S, Pagliari C, Paterson M, Lewis S, et al. Telemonitoring based service redesign for the management of uncontrolled hypertension: multicentre randomised controlled trial. BMJ 2013;346:f3030 [FREE Full text] [Medline: 23709583]

39. Tang PC, Overhage JM, Chan AS, Brown NL, Aghighi B, Entwistle MP, et al. Online disease management of diabetes: engaging and motivating patients online with enhanced resources-diabetes (EMPOWER-D), a randomized controlled trial. J Am Med Inform Assoc 2013 May 1;20(3):526-534 [FREE Full text] [doi: 10.1136/amiajnl-2012-001263] [Medline: 23171659]

40. Svetkey L, Stevens V, Brantley P, Appel L, Hollis J, Loria C, Weight Loss Maintenance Collaborative Research Group. Comparison of strategies for sustaining weight loss: the weight loss maintenance randomized controlled trial. JAMA 2008 Mar 12;299(10):1139-1148. [doi: 10.1001/jama.299.10.1139] [Medline: 18334689]

41. Wing R, Tate D, Gorin A, Raynor H, Fava J. A self-regulation program for maintenance of weight loss. N Engl J Med 2006 Oct 12;355(15):1563-1571. [doi: 10.1056/NEJMoa061883] [Medline: 17035649]

42. Bond G, Burr R, Wolf F, Price M, McCurry S, Teri L. The effects of a web-based intervention on the physical outcomes associated with diabetes among adults age 60 and older: a randomized trial. Diabetes Technol Ther 2007 Feb;9(1):52-59 [FREE Full text] [doi: 10.1089/dia.2006.0057] [Medline: 17316098]

43. Glasgow R, Boles S, McKay H, Feil E, Barrera JM. The D-Net diabetes self-management program: long-term implementation, outcomes, and generalization results. Prev Med 2003 Apr;36(4):410-419. [Medline: 12649049] 
44. Glasgow R, Kurz D, King D, Dickman J, Faber A, Halterman E, et al. Twelve-month outcomes of an Internet-based diabetes self-management support program. Patient Educ Couns 2012 Apr;87(1):81-92 [FREE Full text] [doi: 10.1016/j.pec.2011.07.024] [Medline: 21924576]

45. McMahon G, Gomes H, Hickson HS, Hu T, Levine B, Conlin P. Web-based care management in patients with poorly controlled diabetes. Diabetes Care 2005 Jul;28(7):1624-1629 [FREE Full text] [Medline: 15983311]

46. Avdal E, Kizilci S, Demirel N. The effects of web-based diabetes education on diabetes care results: a randomized control study. Comput Inform Nurs 2011 Feb;29(2):101-106. [doi: 10.1097/NCN.0b013e3181fcbdc6] [Medline: 21099675]

47. Cho J, Chang S, Kwon H, Choi Y, Ko S, Moon S, et al. Long-term effect of the Internet-based glucose monitoring system on HbA1c reduction and glucose stability: a 30-month follow-up study for diabetes management with a ubiquitous medical care system. Diabetes Care 2006 Dec;29(12):2625-2631. [doi: 10.2337/dc05-2371] [Medline: 17130195]

48. Lorig K, Ritter P, Laurent D, Plant K, Green M, Jernigan VB, et al. Online diabetes self-management program: a randomized study. Diabetes Care 2010 Jun;33(6):1275-1281 [FREE Full text] [doi: 10.2337/dc09-2153] [Medline: 20299481]

49. Grant R, Wald J, Schnipper J, Gandhi T, Poon E, Orav E, et al. Practice-linked online personal health records for type 2 diabetes mellitus: a randomized controlled trial. Arch Intern Med 2008 Sep 8;168(16):1776-1782 [FREE Full text] [doi: 10.1001/archinte.168.16.1776] [Medline: 18779465]

50. McMahon G, Fonda S, Gomes H, Alexis G, Conlin P. A randomized comparison of online- and telephone-based care management with internet training alone in adult patients with poorly controlled type 2 diabetes. Diabetes Technol Ther 2012 Nov;14(11):1060-1067 [FREE Full text] [doi: 10.1089/dia.2012.0137] [Medline: 22953754]

51. Ralston J, Hirsch I, Hoath J, Mullen M, Cheadle A, Goldberg H. Web-based collaborative care for type 2 diabetes: a pilot randomized trial. Diabetes Care 2009 Feb;32(2):234-239 [FREE Full text] [doi: 10.2337/dc08-1220] [Medline: 19017773]

52. Kwon H, Cho J, Kim HS, Song BR, Ko SH, Lee JM, et al. Establishment of blood glucose monitoring system using the internet. Diabetes Care 2004 Feb;27(2):478-483. [Medline: 14747232]

53. Fisher L, Hessler D, Glasgow RE, Arean PA, Masharani U, Naranjo D, et al. REDEEM: a pragmatic trial to reduce diabetes distress. Diabetes Care 2013 Sep;36(9):2551-2558 [FREE Full text] [doi: 10.2337/dc12-2493] [Medline: 23735726]

54. Nolan R, Liu S, Shoemaker J, Hachinski V, Lynn H, Mikulis D, et al. Therapeutic benefit of internet-based lifestyle counselling for hypertension. Can J Cardiol 2012 May;28(3):390-396. [doi: 10.1016/j.cjca.2012.02.012] [Medline: 22498181]

55. Bove AA, Homko CJ, Santamore WP, Kashem M, Kerper M, Elliott DJ. Managing hypertension in urban underserved subjects using telemedicine--a clinical trial. Am Heart J 2013 Apr;165(4):615-621. [doi: 10.1016/j.ahj.2013.01.004] [Medline: 23537980]

56. Madsen LB, Kirkegaard P, Pedersen EB. Blood pressure control during telemonitoring of home blood pressure. A randomized controlled trial during 6 months. Blood Press 2008;17(2):78-86. [doi: 10.1080/08037050801915468] [Medline: 18568696]

57. Magid DJ, Olson KL, Billups SJ, Wagner NM, Lyons EE, Kroner BA. A pharmacist-led, American Heart Association Heart360 Web-enabled home blood pressure monitoring program. Circ Cardiovasc Qual Outcomes 2013 Mar 1;6(2):157-163 [FREE Full text] [doi: 10.1161/CIRCOUTCOMES.112.968172] [Medline: 23463811]

58. Thiboutot J, Sciamanna CN, Falkner B, Kephart DK, Stuckey HL, Adelman AM, et al. Effects of a web-based patient activation intervention to overcome clinical inertia on blood pressure control: cluster randomized controlled trial. J Med Internet Res 2013;15(9):e158 [FREE Full text] [doi: 10.2196/jmir.2298] [Medline: 24004475]

59. Bennett G, Herring S, Puleo E, Stein E, Emmons K, Gillman M. Web-based weight loss in primary care: a randomized controlled trial. Obesity (Silver Spring) 2010 Feb;18(2):308-313 [FREE Full text] [doi: 10.1038/oby.2009.242] [Medline: 19696764]

60. Kraschnewski J, Stuckey H, Rovniak L, Lehman E, Reddy M, Poger J, et al. Efficacy of a weight-loss website based on positive deviance. A randomized trial. Am J Prev Med 2011 Dec;41(6):610-614. [doi: 10.1016/j.amepre.2011.08.012] [Medline: 22099238]

61. Webber KH, Tate DF, Michael BJ. A randomized comparison of two motivationally enhanced Internet behavioral weight loss programs. Behav Res Ther 2008 Sep;46(9):1090-1095. [doi: 10.1016/j.brat.2008.06.008] [Medline: 18675402]

62. Yardley L, Ware LJ, Smith ER, Williams S, Bradbury KJ, Arden-Close EJ, et al. Randomised controlled feasibility trial of a web-based weight management intervention with nurse support for obese patients in primary care. Int J Behav Nutr Phys Act 2014;11:67 [FREE Full text] [doi: 10.1186/1479-5868-11-67] [Medline: 24886516]

63. Richardson C, Buis L, Janney A, Goodrich D, Sen A, Hess M, et al. An online community improves adherence in an internet-mediated walking program. Part 1: results of a randomized controlled trial. J Med Internet Res 2010;12(4):e71 [FREE Full text] [doi: 10.2196/jmir.1338] [Medline: 21169160]

64. Ferney S, Marshall A, Eakin E, Owen N. Randomized trial of a neighborhood environment-focused physical activity website intervention. Prev Med 2009 Feb;48(2):144-150. [doi: 10.1016/j.ypmed.2008.10.022] [Medline: 19028519]

65. Irvine AB, Gelatt VA, Seeley JR, Macfarlane P, Gau JM. Web-based intervention to promote physical activity by sedentary older adults: randomized controlled trial. J Med Internet Res 2013;15(2):e19 [FREE Full text] [doi: 10.2196/jmir.2158] [Medline: 23470322]

66. Wijsman CA, Westendorp RG, Verhagen EA, Catt M, Slagboom PE, de Craen AJ, et al. Effects of a web-based intervention on physical activity and metabolism in older adults: randomized controlled trial. J Med Internet Res 2013;15(11):e233 [FREE Full text] [doi: 10.2196/jmir.2843] [Medline: 24195965] 
67. Suboc TB, Strath SJ, Dharmashankar K, Coulliard A, Miller N, Wang J, et al. Relative importance of step count, intensity, and duration on physical activity's impact on vascular structure and function in previously sedentary older adults. J Am Heart Assoc 2014;3(1):e000702 [FREE Full text] [doi: 10.1161/JAHA.113.000702] [Medline: 24572255]

68. Peels DA, Bolman C, Golsteijn RH, de Vries H, Mudde AN, van Stralen MM, et al. Long-term efficacy of a printed or a Web-based tailored physical activity intervention among older adults. Int J Behav Nutr Phys Act 2013;10:104 [FREE Full text] [doi: 10.1186/1479-5868-10-104] [Medline: 24228862]

69. Bloch MJ, Armstrong DS, Dettling L, Hardy A, Caterino K, Barrie S. Partners in lowering cholesterol: comparison of a multidisciplinary educational program, monetary incentives, or usual care in the treatment of dyslipidemia identified among employees. J Occup Environ Med 2006 Jul;48(7):675-681. [doi: 10.1097/01.jom.0000205997.18143.6c] [Medline: 16832224]

70. Kulick D, Langer RD, Ashley JM, Gans KM, Schlauch K, Feller C. Live well: a practical and effective low-intensity dietary counseling intervention for use in primary care patients with dyslipidemia--a randomized controlled pilot trial. BMC Fam Pract 2013;14:59 [FREE Full text] [doi: 10.1186/1471-2296-14-59] [Medline: 23663789]

71. Lindsay S, Bellaby P, Smith S, Baker R. Enabling healthy choices: is ICT the highway to health improvement? Health (London) 2008 Jul;12(3):313-331. [doi: 10.1177/1363459308090051] [Medline: 18579630]

72. Brennan PF, Casper GR, Burke LJ, Johnson KA, Brown R, Valdez RS, et al. Technology-enhanced practice for patients with chronic cardiac disease: home implementation and evaluation. Heart Lung 2010;39(6 Suppl):S34-546 [FREE Full text] [doi: 10.1016/j.hrtlng.2010.09.003] [Medline: 21092830]

73. Hughes S, Seymour R, Campbell R, Shaw J, Fabiyi C, Sokas R. Comparison of two health-promotion programs for older workers. Am J Public Health 2011 May;101(5):883-890 [FREE Full text] [doi: 10.2105/AJPH.2010.300082] [Medline: $\underline{21421955]}$

74. Winett R, Anderson E, Wojcik J, Winett S, Bowden T. Guide to health: nutrition and physical activity outcomes of a group-randomized trial of an Internet-based intervention in churches. Ann Behav Med 2007 Jun;33(3):251-261. [doi: 10.1080/08836610701358045] [Medline: 17600452]

75. Verheijden M, Bakx J, Akkermans R, van den Hoogen H, Godwin N, Rosser W, et al. Web-based targeted nutrition counselling and social support for patients at increased cardiovascular risk in general practice: randomized controlled trial. J Med Internet Res 2004 Dec 16;6(4):e44 [FREE Full text] [doi: 10.2196/jmir.6.4.e44] [Medline: 15631968]

76. Bove A, Santamore W, Homko C, Kashem A, Cross R, McConnell T, et al. Reducing cardiovascular disease risk in medically underserved urban and rural communities. Am Heart J 2011 Feb;161(2):351-359. [doi: 10.1016/j.ahj.2010.11.008] [Medline: 21315219]

77. Zullig LL, Sanders LL, Shaw RJ, McCant F, Danus S, Bosworth HB. A randomised controlled trial of providing personalised cardiovascular risk information to modify health behaviour. J Telemed Telecare 2014 Apr;20(3):147-152. [doi: 10.1177/1357633X14528446] [Medline: 24647384]

78. Devi R, Powell J, Singh S. A web-based program improves physical activity outcomes in a primary care angina population: randomized controlled trial. J Med Internet Res 2014;16(9):e186 [FREE Full text] [doi: 10.2196/jmir.3340] [Medline: 25217464]

79. Green BB, Anderson ML, Cook AJ, Catz S, Fishman PA, McClure JB, et al. e-Care for heart wellness: a feasibility trial to decrease blood pressure and cardiovascular risk. Am J Prev Med 2014 Apr;46(4):368-377 [FREE Full text] [doi: 10.1016/j.amepre.2013.11.009] [Medline: 24650839]

80. Greene J, Sacks R, Piniewski B, Kil D, Hahn JS. The impact of an online social network with wireless monitoring devices on physical activity and weight loss. J Prim Care Community Health 2013 Jul 1;4(3):189-194. [doi:

10.1177/2150131912469546] [Medline: 23799706]

81. Holbrook A, Thabane L, Keshavjee K, Dolovich L, Bernstein B, Chan D, et al. Individualized electronic decision support and reminders to improve diabetes care in the community: COMPETE II randomized trial. CMAJ 2009 Jul 7;181(1-2):37-44 [FREE Full text] [doi: 10.1503/cmaj.081272] [Medline: 19581618]

82. Jennings CA, Vandelanotte C, Caperchione CM, Mummery WK. Effectiveness of a web-based physical activity intervention for adults with Type 2 diabetes-a randomised controlled trial. Prev Med 2014 Mar;60:33-40. [doi:

10.1016/j.ypmed.2013.12.011] [Medline: 24345601]

83. Appel LJ, Brands MW, Daniels SR, Karanja N, Elmer PJ, Sacks FM, et al. Dietary approaches to prevent and treat hypertension: a scientific statement from the American Heart Association. Hypertension 2006 Feb;47(2):296-308 [FREE Full text] [doi: 10.1161/01.HYP.0000202568.01167.B6] [Medline: 16434724]

84. Aalbers T, Baars MA, Rikkert MG. Characteristics of effective Internet-mediated interventions to change lifestyle in people aged 50 and older: a systematic review. Ageing Res Rev 2011 Sep;10(4):487-497. [doi: 10.1016/j.arr.2011.05.001] [Medline: 21628005]

85. Schwingshackl L, Dias S, Hoffmann G. Impact of long-term lifestyle programmes on weight loss and cardiovascular risk factors in overweight/obese participants: a systematic review and network meta-analysis. Syst Rev 2014;3:130 [FREE Full text] [doi: 10.1186/2046-4053-3-130] [Medline: 25358395]

86. Mellen PB, Gao SK, Vitolins MZ, Goff DC. Deteriorating dietary habits among adults with hypertension: DASH dietary accordance, NHANES 1988-1994 and 1999-2004. Arch Intern Med 2008 Feb 11;168(3):308-314. [doi:

10.1001/archinternmed.2007.119] [Medline: 18268173] 
87. Fjeldsoe B, Neuhaus M, Winkler E, Eakin E. Systematic review of maintenance of behavior change following physical activity and dietary interventions. Health Psychol 2011 Jan;30(1):99-109. [doi: 10.1037/a0021974] [Medline: 21299298]

88. Ebrahim S, Taylor F, Ward K, Beswick A, Burke M, Davey SG. Multiple risk factor interventions for primary prevention of coronary heart disease. Cochrane Database Syst Rev 2011(1):CD001561. [doi: 10.1002/14651858.CD001561.pub3] [Medline: 21249647]

89. Fifty-eighth World Health Assembly, Geneva, 16-25 May 2005. Geneva: World Health Organization; 2005. Resolutions and decisions: eHealth (WHA58/2005/REC/1) URL: http://www.who.int/healthacademy/media/WHA58-28-en.pdf [accessed 2015-10-08] [WebCite Cache ID 6c8Lz3pCS]

90. Kroes N. European Commission. 2014 May 12. Shaping a healthier, stronger future URL: http://europa.eu/rapid/ press-release_SPEECH-14-369_en.htm[WebCite Cache ID 6fU5p79na]

\author{
Abbreviations \\ BP: blood pressure \\ HbA1c: glycated hemoglobin A1c \\ IQR: interquartile range \\ LDL: low-density lipoprotein \\ PA: physical activity \\ RCT: randomized controlled trial
}

Edited by G Eysenbach; submitted 12.10.15; peer-reviewed by B Green, S Anand, A Burdorf, R Widmer; comments to author 22.11.15;
revised version received 18.12.15; accepted 17.01.16; published 11.03.16
Please cite as:
Beishuizen CRL, Stephan BCM, van Gool WA, Brayne C, Peters RJG, Andrieu S, Kivipelto M, Soininen H, Busschers WB, Moll van
Charante EP, Richard E
Web-Based Interventions Targeting Cardiovascular Risk Factors in Middle-Aged and Older People: A Systematic Review and
Meta-Analysis
JMed Internet Res 2016;18(3):e55
URL: http://www.jmir.org/2016/3/e55/
doi: $\underline{10.2196 / j m i r .5218}$
PMID: $\underline{26968879}$

CCathrien RL Beishuizen, Blossom CM Stephan, Willem A van Gool, Carol Brayne, Ron JG Peters, Sandrine Andrieu, Miia Kivipelto, Hilkka Soininen, Wim B Busschers, Eric P Moll van Charante, Edo Richard. Originally published in the Journal of Medical Internet Research (http://www.jmir.org), 11.03.2016. This is an open-access article distributed under the terms of the Creative Commons Attribution License (http://creativecommons.org/licenses/by/2.0/), which permits unrestricted use, distribution, and reproduction in any medium, provided the original work, first published in the Journal of Medical Internet Research, is properly cited. The complete bibliographic information, a link to the original publication on http://www.jmir.org/, as well as this copyright and license information must be included. 